\title{
Semi-inclusive $B$ decays and a model for soft-gluon effects
}

\author{
Ugo Aglietti a , Giancarlo Ferrera ${ }^{\text {a }}$, Giulia Ricciardi ${ }^{\mathrm{b}, *}$ \\ a Dipartimento di Fisica, Università di Roma "La Sapienza" and INFN, Sezione di Roma, Italy \\ b Dipartimento di Scienze Fisiche, Università di Napoli "Federico II" and INFN, Sezione di Napoli, Italy
}

Received 5 August 2006; received in revised form 21 November 2006; accepted 8 January 2007

Available online 20 January 2007

\begin{abstract}
We compare experimental spectra of radiative and semileptonic $B$ decays with the predictions of a model based on soft-gluon resummation to next-to-next-to-leading order and on a ghost-less time-like coupling. We find a good agreement with photon spectra in the radiative decay and with hadron mass distributions in the semileptonic one: the extracted values for $\alpha_{S}\left(m_{Z}\right)$ are in agreement with the current PDG average within at most two standard deviations. The agreement is instead less good for the electron spectra measured by BaBar and Belle in semileptonic decays for small electron energies $(\leqslant 2.2 \mathrm{GeV})$ : our spectrum is harder. We also show that, in general, the inclusion of next-to-next-to-leading order effects is crucial for bringing the model closer to the data and that the non-power expansion introduced in the framework of analytic coupling studies does not accurately describe soft-gluon effects.
\end{abstract}

(C) 2007 Elsevier B.V. All rights reserved.

\section{Introduction}

The aim of this work is to analyze measured $B$ decay spectra with a model based on (i) soft gluon resummation to next-to-next-to-leading order and (ii) an effective QCD coupling having no Landau pole [1]. This coupling is constructed by means of an extrapolation at low energy of the high-energy behavior of the standard coupling. More technically, an analyticity principle is used.

\footnotetext{
* Corresponding author.

E-mail addresses: ugo.aglietti@ roma1.infn.it (U. Aglietti), giancarlo.ferrera@ roma1.infn.it (G. Ferrera), giulia.ricciardi@na.infn.it (G. Ricciardi).
} 
$B$ decay spectra are substantially affected by long-distance effects, the most important ones being the soft interactions occurring in the fragmentation of the $B$ meson into the $b$ quark. The $B$ meson-a colorless composite particle-emits the spectator quark and radiates soft gluons,

$$
B \rightarrow b+s p+g_{1}+g_{2}+\cdots+g_{n},
$$

to convert into the colored $b$ quark which later decays because of weak interactions,

$$
b \rightarrow s+\gamma
$$

or

$$
b \rightarrow u+l+v .
$$

Perturbation theory can describe the fragmentation of a $b$ quark into a $b$ quark with a fraction of the original energy-momentum as an effect of multiple gluon radiation, but it clearly cannot describe that part of the fragmentation involving the spectator quark. Physical intuition suggests that initial bound-state effects are substantial for

$$
m_{X}^{2} \approx m_{B} \Lambda_{\mathrm{QCD}} \approx 2 \mathrm{GeV}^{2},
$$

which is experimentally interesting: that is the well-known Fermi motion of the $b$ quark in the $B$ meson ( $m_{X}$ is the final invariant hadron mass). This non-perturbative effect-which classically can be pictured as a small vibration of the $b$ quark in the $B$ meson because of the interactions with the spectator-has been formalized in an effective field theory by means of the well-known shape function or structure function of the heavy flavors [2]. Many models have been constructed to describe Fermi-motion as a genuinely non-perturbative effect involving the hadron structure [3]; perturbative corrections are included, if desired, later on and play in any case a minor role. In this work we adopt a different philosophy: in essence, we assume that the fragmentation of the lowest-lying beauty meson into the beauty quark and the spectator quark can be described as a radiation process off the $b$ with a proper coupling. Even though dynamics of light degrees of freedom in the $B$ meson is complicated, we assume that the related effects on semi-inclusive spectra are simple. More precisely, we assume that bound-state effects can be incorporated into an effective QCD coupling, which is inserted in the standard soft-gluon resummation formulas. We extrapolate therefore the perturbative QCD formulas to a non-perturbative region by assuming that the relevant non-perturbative effects can be relegated into an effective coupling. Since the perturbative formulas involve truncated expansions in the QCD coupling, it is clear that our approach is meaningful as long as the effective coupling remains appreciably smaller than one in all the relevant integration range. From Fig. 2 we see that our effective coupling is $\approx 0.5$ for a typical soft scale $k_{\perp} \approx 0.5 \mathrm{GeV}$ (corresponding to $x_{\gamma}=2 E_{\gamma} / m_{B} \approx 0.9$ in radiative $B$ decays), i.e. it is reasonably smaller than one.

Since the whole fragmentation process is described in a perturbative framework, we do not distinguish between the mass of the $B$ meson and the pole mass of the $b$ quark, i.e. we consistently set $m_{b}=m_{B}$. We also assume that this effective coupling is universal, i.e. that it can be used to describe different processes, and that it can be constructed on the basis of analyticity arguments. These are additional assumptions with respect to the basic one, which could eventually be relaxed.

Let us remark that the resummed perturbative expansion for semi-inclusive quantities is incomplete even at the formal level. For inclusive quantities characterized by a hard scale $Q$, the 
cross-section can be written in a consistent way as an expansion in the coupling at the scale $Q$,

$$
\sigma_{\text {incl }}(Q)=\sum_{n=0}^{\infty} c_{n} \alpha_{S}^{n}\left(Q^{2}\right),
$$

where the $c_{n}$ 's are numerical coefficients of order one: no prescription is needed. Semi-inclusive processes are instead multi-scale processes, characterized by fluctuations with transverse momenta $u p$ to $Q$; the physical origin is very clear: a jet with a relatively large invariant mass $m_{X}$ $\left(\Lambda_{\mathrm{QCD}} \ll m_{X} \ll Q\right)$ can contain very soft partons, with transverse momenta of the order of the hadronic scale. Unlike case (5), one has to face perturbative contributions of the form

$$
\int_{\approx 0}^{Q^{2}} \frac{d k_{\perp}^{2}}{k_{\perp}^{2}} \alpha_{S}\left(k_{\perp}^{2}\right),
$$

where an ill-defined integration over the Landau pole is made, even for large $Q \gg \Lambda_{\mathrm{QCD}}$. A prescription for the low-energy behavior of the coupling is therefore needed in any case. Even if quark confinement did not exist and partons instead of hadrons were the asymptotic states, a prescription would anyway be necessary to compute resummed cross-sections.

It is clear that our approach has intrinsic and obvious limitations. The mass of the proton, for example, cannot clearly be computed by means of perturbative formulas with an effective coupling inserted in them: a genuinely non-perturbative technique is mandatory in this case, such as lattice QCD. Our point is that, with an effective coupling, we want to describe Fermi motion only, i.e. a specific non-perturbative effect, not all non-perturbative effects. We do not aim for example at describing the $K^{*}$ peak which appears in the radiative hadron mass distribution (see Fig. 3), or, equivalently, the $\pi$ and $\rho$ peaks which appear in the semileptonic one (see Figs. 7 and 8). These peaks, occurring for

$$
m_{X}^{2} \approx \Lambda_{\mathrm{QCD}}^{2}
$$

are related to final-state hadronization, i.e. to the recombination of partons into hadrons. This effect has a different nature with respect to Fermi motion and occurs at a different scale (cf. Eq. (4) with Eq. (7)). With our model, we just want to describe a broad peak in the hadron mass distribution occurring in region (4). A possible difference between the photon spectra of, let us say, $B \rightarrow X_{s} \gamma$ and $\Lambda_{b} \rightarrow X_{s} \gamma$ decays, could not be described or naturally incorporated in our model, which is a kind of "spectator model for spectra".

The validity of our approach cannot be judged a priori, but only a posteriori, by comparing its predictions with experimental data. One may ask which is the advantage of our approach compared to the standard one of postulating directly shapes for the non-perturbative components of the spectra and convolving them with the perturbative ones in the minimal prescription [4]. The answer is that we want to take advantage of the universality properties of QCD radiation, which are reflected in resummation formulas. In the standard approach, one has to postulate ad-hoc and un-related shapes for the non-perturbative components entering different observables, such as heavy flavor decay spectra, heavy flavor fragmentation, $e^{+} e^{-}$shape variables, etc. If universal aspects of QCD dynamics — as measured in different processes-do exist, such aspects are not easily uncovered with the standard approach. On the other hand, with our method, such an investigation looks rather natural: to describe different processes, we use different perturbative formulas - quite often the same formulas but with different coefficients-with the same effective coupling by assumption and we look at the data [5]. Our philosophy involves a "one step" 
approach: we deal simultaneously with perturbative and non-perturbative effects. The standard method is instead a "two-step" approach: one resums the perturbative long-distance effects in a minimal way - picking up just the infrared logarithms - and then introduces a physically motivated non-perturbative model.

Another advantage of our approach is that it allows for a simple extraction of the value of the standard QCD coupling at a reference scale, f.i. $\alpha_{S}\left(m_{Z}\right)$, by comparing its predictions with measured $B$ decay spectra. That is because the model uses ordinary perturbative formulas with a prescription for the coupling in the low-energy tail, and therefore there is not any double-counting problem in merging together short-distance and long-distance effects. A peculiarity of our model is that it has no free parameters, apart of course the true QCD ones, i.e. the hadronic scale $\Lambda_{\mathrm{QCD}}$ and the quark masses $m_{q}$ 's. It is therefore "rigid", in the sense that there is not a natural way to tune it to fit the data.

The plan of the paper is as follows.

In Section 2 we summarize the main features of the ghost-less QCD coupling, which is basically an extrapolation of the ordinary QCD coupling down to small momentum scales according to an analyticity principle which removes the Landau pole.

In Section 3 we construct the effective coupling controlling the evolution of gluon cascades, which are intrinsically time-like processes. The absorptive effects related to the decay of the time-like gluons are included in this effective coupling to all orders in perturbation theory.

Section 4 is the main one and describes the model based on soft gluon resummation in NNLO and on the effective coupling constructed in the previous section. A discussion of the relevance of the next-to-next-to-leading-order effects in our model is also presented. We also comment on the non-power expansion introduced in analytic coupling studies.

In Section 5 we apply the model to describe $B \rightarrow X_{s} \gamma$ decays. We compare its predictions with the invariant hadron mass distribution measured by BaBar and with the inclusive photon spectrum measured by CLEO, BaBar and Belle. Since these spectra are independent from each other, we obtain for each of them a value of $\alpha_{S}\left(m_{Z}\right)$ which optimizes the agreement with the data.

In Section 6 we apply the model to the charmless semileptonic decays $B \rightarrow X_{u} l v$. We compare our predictions with the invariant hadron mass distribution measured by BaBar and Belle and with the charged lepton energy spectrum measured by CLEO, BaBar and Belle. We extract values of $\alpha_{S}\left(m_{Z}\right)$ as discussed above.

Finally, in Section 7 we draw our conclusions concerning the agreement of the model with the data. We also consider natural developments and improvements.

There is also an appendix collecting formulas for the radiative decay and an appendix with tables of values of the QCD form factor in our model for a set of values of $\alpha_{S}\left(m_{Z}\right)$.

\section{Ghost-less coupling}

Let us begin considering QCD regularized with an ultra-violet cut-off $\Lambda_{0}$ and with a bare coupling $\alpha_{0}$. The correlation function ${ }^{1}$ representing the quark-gluon interaction has a perturbative expansion of the form:

\footnotetext{
1 To be accurate, we consider the $q \bar{q} g$ correlation function amputated of all legs and written in terms of the renormalized fields.
} 


$$
\begin{aligned}
& \Gamma_{q \bar{q} g}\left(p_{1}^{2}=p_{2}^{2}=p_{3}^{2}=q^{2}\right) \\
& \quad=\alpha_{0}+\beta_{0} \alpha_{0}^{2} \log \frac{\Lambda_{0}^{2}}{-q^{2}-i \varepsilon}+\alpha_{0}^{2} c+\beta_{0}^{2} \alpha_{0}^{3} \log ^{2} \frac{\Lambda_{0}^{2}}{-q^{2}-i \varepsilon}+\cdots \\
& \quad=\frac{\alpha_{0}}{1-\beta_{0} \alpha_{0} \log \Lambda_{0}^{2} /\left(-q^{2}-i \varepsilon\right)}+\cdots,
\end{aligned}
$$

where for simplicity's sake we have considered the symmetric point $p_{1}^{2}=p_{2}^{2}=p_{3}^{2}$. In the last member we have resummed the well-known geometrical series of the leading logarithms. $\beta_{0}=$ $\left(11-2 / 3 n_{f}\right) /(4 \pi)$ is the first-coefficient of the $\beta$-function, $n_{f}$ is the number of active flavors and $c$ is a real constant whose explicit expression is not relevant here. This Green function can be used to define the renormalized QCD coupling [6]:

$$
\Gamma_{q \bar{q} g}\left(p_{1}^{2}=p_{2}^{2}=p_{3}^{2}=q^{2}\right) \simeq \frac{\alpha_{0}}{1-\beta_{0} \alpha_{0} \log \Lambda_{0}^{2} /\left(-q^{2}-i \varepsilon\right)} \equiv \alpha\left(-q^{2}\right) .
$$

To have a real coupling, one generally assumes a space-like configuration of the momenta,

$$
q^{2}<0
$$

and to avoid explicit minus signs in the renormalization conditions, one defines, like in DeepInelastic-Scattering (DIS $)^{2}$ :

$$
Q^{2} \equiv-q^{2} \text {. }
$$

We then obtain the usual expression for the renormalized QCD coupling in leading order (LO):

$$
\alpha_{\mathrm{lo}}\left(Q^{2}\right)=\frac{\alpha_{0}}{1-\beta_{0} \alpha_{0} \log \Lambda_{0}^{2} / Q^{2}}=\frac{1}{\beta_{0} \log Q^{2} / \Lambda_{\mathrm{QCD}}^{2}},
$$

where on the last member we have introduced the QCD scale

$$
\Lambda_{\mathrm{QCD}}^{2} \equiv \Lambda_{0}^{2} \exp \left[-\frac{1}{\beta_{0} \alpha_{0}}\right] \text {. }
$$

For notational simplicity, let us write $\Lambda$ in place of $\Lambda_{\mathrm{QCD}}$ from now on. The function on the r.h.s. of Eq. (13) has:

1. a cut for $Q^{2}<0^{3}$, related to the decay of a time-like gluon into secondary partons,

$$
g^{*} \rightarrow g g, q \bar{q}, \ldots
$$

This singularity has therefore a clear physical meaning;

2. a simple pole for $Q^{2}=\Lambda^{2}$, which does not have any physical meaning [7]. This singularity is often called "Landau ghost" because of its original appearance in QED in the interacting electron propagator [8]. It implies a formal divergence of the coupling and a breakdown of the perturbative scheme.

$\overline{{ }^{2} \text { Note that }} Q^{2}>0$ in the space-like region while $Q^{2}<0$ in the time-like one and the $\varepsilon$-prescription for $Q^{2}$ is opposite to that for $q^{2}$ :

$$
Q^{2} \equiv Q^{2}-i \varepsilon
$$

3 As usual, the logarithm function is cut along the negative semi-axis, so that: $\log (-1 \pm i \varepsilon)= \pm i \pi$. 


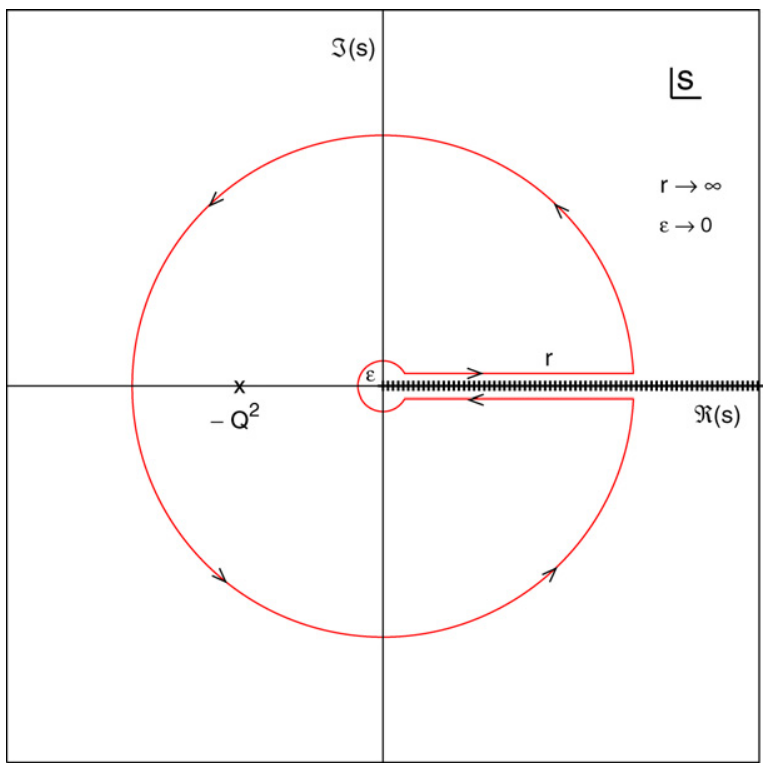

Fig. 1. Integration contour $\Gamma$ used to construct the ghost-less coupling.

It has been suggested to replace the usual expression for the coupling in Eq. (13) with a "ghostless" or "analytic" coupling $\bar{\alpha}$ having the following properties [1]:

1. it has the same discontinuity along the cut as the standard coupling:

$\operatorname{Disc} \bar{\alpha}=\operatorname{Disc} \alpha \quad$ (time-like region);

2. it is analytic elsewhere in the complex plane.

Let us now consider the function

$$
f(s) \equiv \frac{\bar{\alpha}(-s)}{s+Q^{2}},
$$

where $Q^{2}$ is a complex number not lying on the negative axis including the origin. By assumption, $f(s)$ is analytic in the complex $s$-plane cut along the positive axis $s \geqslant 0$, except for a simple pole in

$$
s=-Q^{2}
$$

We apply the residue theorem to $f(s)$ integrated along a closed contour $\Gamma$ avoiding the "physical" cut for $s \geqslant 0$, containing a circle of infinitesimal radius around the origin $c_{\epsilon}(\epsilon \rightarrow 0)$, a circle at infinity $c_{r}(r \rightarrow \infty)$, a line above the cut $(s \rightarrow s+i \varepsilon)$ and a line below the cut $(s \rightarrow s-i \varepsilon)$ : see Fig. 1. Being the pole (18) the only singularity inside the contour, we obtain the following 
expression for the analytic coupling ${ }^{4}$ :

$$
\begin{aligned}
\bar{\alpha}\left(Q^{2}\right)= & \frac{1}{2 \pi i} \oint_{\Gamma} \frac{\bar{\alpha}(-s)}{s+Q^{2}} d s \\
= & \frac{1}{2 \pi i} \oint_{c_{\epsilon}} \frac{\bar{\alpha}(-s)}{s+Q^{2}} d s+\frac{1}{2 \pi i} \oint_{c_{r}} \frac{\bar{\alpha}(-s)}{s+Q^{2}} d s \\
& +\frac{1}{2 \pi i} \int_{0}^{\infty} \frac{\bar{\alpha}(-s-i \varepsilon)}{s+i \varepsilon+Q^{2}} d s+\frac{1}{2 \pi i} \int_{\infty}^{0} \frac{\bar{\alpha}(-s+i \varepsilon)}{s-i \varepsilon+Q^{2}} d s .
\end{aligned}
$$

We assume that the contributions of $c_{\epsilon}$ and of $c_{r}$ vanish. Since $s+Q^{2} \neq 0$ for $s \geqslant 0$,

$$
\begin{aligned}
\lim _{\varepsilon \rightarrow 0^{+}}\left[\frac{\bar{\alpha}(-s-i \varepsilon)}{s+i \varepsilon+Q^{2}}-\frac{\bar{\alpha}(-s+i \varepsilon)}{s-i \varepsilon+Q^{2}}\right] & =\frac{1}{s+Q^{2}} \lim _{\varepsilon \rightarrow 0^{+}}[\bar{\alpha}(-s-i \varepsilon)-\bar{\alpha}(-s+i \varepsilon)] \\
& =\frac{1}{s+Q^{2}} \operatorname{Disc}_{s} \bar{\alpha}(-s),
\end{aligned}
$$

where the discontinuity of a function $F(s)$ is defined in general as:

$$
\operatorname{Disc}_{s} F(s) \equiv \lim _{\varepsilon \rightarrow 0^{+}}[F(s+i \varepsilon)-F(s-i \varepsilon)] .
$$

Taking into account that for $s \geqslant 0$ (see Eq. (16))

$$
\operatorname{Disc}_{s} \bar{\alpha}(-s)=\operatorname{Disc}_{s} \alpha(-s),
$$

we obtain the following integral representation for the ghost-less coupling in terms of the standard one:

$$
\bar{\alpha}\left(Q^{2}\right)=\frac{1}{2 \pi i} \int_{0}^{\infty} \frac{d s}{s+Q^{2}} \operatorname{Disc}_{s} \alpha(-s) .
$$

Eq. (23) is just a dispersion relation which, for clarity's sake, has been fully derived from first principles. By inserting on the last member the expression for the standard coupling at lowest order as given by Eq. (13), we obtain:

$$
\bar{\alpha}_{\mathrm{lo}}\left(Q^{2}\right)=\lim _{\varepsilon \rightarrow 0^{+}} \frac{1}{2 \pi i \beta_{0}} \int_{0}^{\infty} \frac{d s}{s+Q^{2}}\left[\frac{1}{\log \left(-s / \Lambda^{2}-i \varepsilon\right)}-\frac{1}{\log \left(-s / \Lambda^{2}+i \varepsilon\right)}\right] .
$$

The integral above is elementary. It can also be computed with the residue theorem by considering the contour $\Gamma$ above. The circle of infinitesimal radius around the origin and the circle at infinity give vanishing contributions to the integral. There are two simple poles in $s=-Q^{2}$ and in $s=-\Lambda^{2}$, so that:

$$
\bar{\alpha}_{\mathrm{lo}}\left(Q^{2}\right)=\frac{1}{\beta_{0}}\left[\frac{1}{\log Q^{2} / \Lambda^{2}}-\frac{\Lambda^{2}}{Q^{2}-\Lambda^{2}}\right] .
$$

Let us make a few remarks:

\footnotetext{
4 Note that, had we taken $Q^{2} \leqslant 0$, the pole (18) would have been located on the cut and the integral of $f(s)$ over $\Gamma$ would have been zero.
} 
1. comparing the r.h.s. of Eqs. (13) and (25), we see that the "analyticization" procedure had the effect of subtracting the infrared pole in $Q^{2}=\Lambda^{2}$ by means of a power-suppressed term, in a minimal way;

2. the analytic coupling has a constant limit at zero momentum transfer:

$$
\lim _{Q^{2} \rightarrow 0} \bar{\alpha}_{\mathrm{lo}}\left(Q^{2}\right)=\frac{1}{\beta_{0}} \approx O(1) ;
$$

3. the term added to the standard coupling,

$$
-\frac{1}{\beta_{0}} \frac{\Lambda^{2}}{Q^{2}-\Lambda^{2}},
$$

does not modify the high-energy behavior because it decays as an inverse power of the hard scale, i.e. infinitely faster than any inverse power of the logarithm of $Q^{2}$. In more formal terms, the added term (27) is exponentially small in the coupling, and therefore is always missed in an asymptotic expansion for $Q^{2} \rightarrow \infty$ :

$$
\frac{\Lambda^{2}}{Q^{2}-\Lambda^{2}}=\frac{1}{e^{1 /\left[\beta_{0} \alpha_{\mathrm{lo}}\left(Q^{2}\right)\right]}-1} \approx e^{-1 /\left[\beta_{0} \alpha_{\mathrm{lo}}\left(Q^{2}\right)\right]} ;
$$

4. since the power correction has no discontinuity in the time-like region $Q^{2}<0$,

$$
\operatorname{Disc} \frac{\Lambda^{2}}{Q^{2}-\Lambda^{2}}=0 \text { for } Q^{2}<0,
$$

it trivially follows that the analytic coupling has the same discontinuity as the standard one, as originally requested.

Let us now discuss the extension to next-to-leading order (NLO). The NLO correction to the standard coupling

$$
\delta \alpha\left(Q^{2}\right)=-\frac{\beta_{1}}{\beta_{0}^{3}} \log \left(\log \frac{Q^{2}}{\Lambda^{2}}\right) \frac{1}{\log ^{2} Q^{2} / \Lambda^{2}},
$$

where $\beta_{1}$ is the second-order coefficient of the $\beta$-function in the normalization assumed in [9], involves:

1. the factor $1 / \log ^{2} Q^{2} / \Lambda^{2}$, having a cut for $Q^{2}<0$, related to the decay of the time-like gluon into on-shell partons (see Eq. (15)), and a double pole for $Q^{2}=\Lambda^{2}$;

2. the factor $\log \left(\log Q^{2} / \Lambda^{2}\right)$, having a cut for $Q^{2}<0$ related to the "internal" logarithm and another cut for $0<Q^{2}<\Lambda^{2}$ related to the "external" logarithm.

The singularities for $Q^{2}=\Lambda^{2}$ and for $0<Q^{2}<\Lambda^{2}$ are unphysical because they refer to the space-like region, where the virtual gluon cannot decay into physical parton states. "Analyticization" can be made as in lowest order: one requires that the analytic correction term has the same discontinuity for $Q^{2}<0$ as the standard one but it is regular elsewhere in the complex plane:

$$
\delta \bar{\alpha}\left(Q^{2}\right)=\frac{1}{2 \pi i} \int_{0}^{\infty} \frac{d s}{s+Q^{2}} \operatorname{Disc}_{s} \delta \alpha(-s) .
$$


The integral above-unlike the lowest-order case - is not elementary but it can easily be made numerically. The following remarks are in order:

- the value of the analytic coupling at zero momentum transfer is not modified in higher order because:

$$
\lim _{Q^{2} \rightarrow 0} \delta \bar{\alpha}\left(Q^{2}\right)=0
$$

- it can be shown that $\delta \bar{\alpha}\left(Q^{2}\right)$ has the same logarithmic terms as $\delta \alpha\left(Q^{2}\right)$ [10], i.e. that the difference resides in power-suppressed terms, as we have explicitly found for the leading order.

The NLO coupling is defined as: $\bar{\alpha}_{\mathrm{nl}}=\bar{\alpha}_{\mathrm{lo}}+\delta \bar{\alpha}$. Within our accuracy, the next-to-next-to-leading order (NNLO) corrections to the coupling are also needed:

$$
\delta \alpha^{\prime}\left(Q^{2}\right)=\frac{\beta_{1}^{2}}{\beta_{0}^{5}}\left[\log ^{2}\left(\log \frac{Q^{2}}{\Lambda^{2}}\right)-\log \left(\log \frac{Q^{2}}{\Lambda^{2}}\right)+\frac{\beta_{0} \beta_{2}-\beta_{1}^{2}}{\beta_{1}^{2}}\right] \frac{1}{\log ^{3} Q^{2} / \Lambda^{2}},
$$

where $\beta_{2}$ is the third-order coefficient of the $\beta$-function. One finds similar singularities as in the NLO case, which are removed again according to principle of "minimal analyticity" already used:

$$
\delta \bar{\alpha}^{\prime}\left(Q^{2}\right)=\frac{1}{2 \pi i} \int_{0}^{\infty} \frac{d s}{s+Q^{2}} \operatorname{Disc}_{s} \delta \alpha^{\prime}(-s) .
$$

The NNLO analytic coupling reads (see Fig. 2):

$$
\bar{\alpha}=\bar{\alpha}_{\mathrm{lo}}+\delta \bar{\alpha}+\delta \bar{\alpha}^{\prime} \text {. }
$$

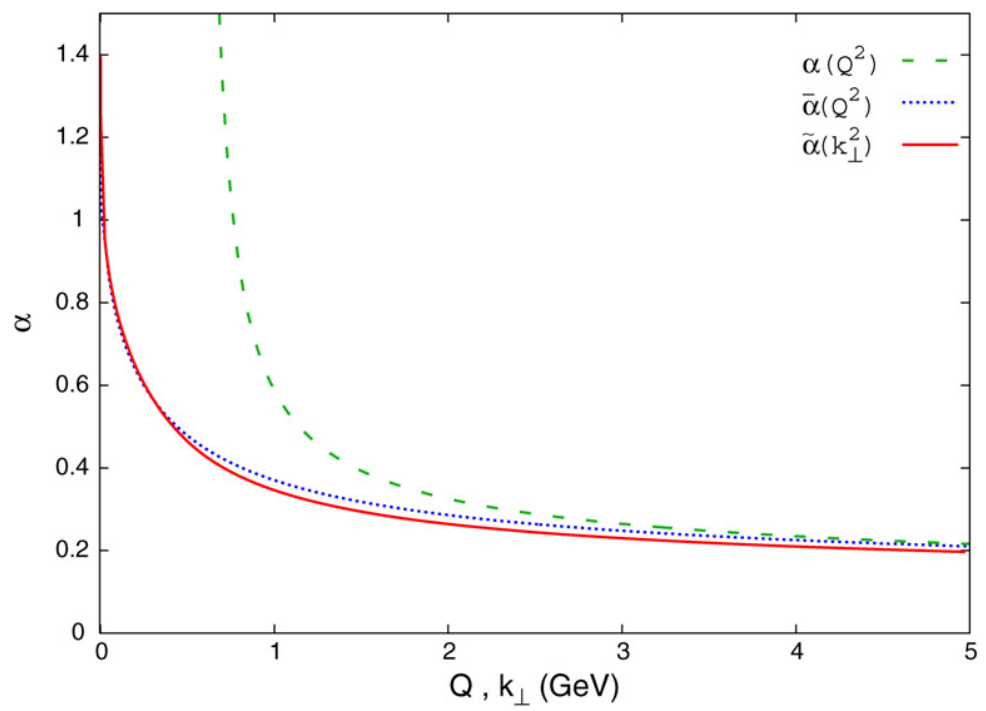

Fig. 2. QCD couplings in NNLO for a fixed number of active flavors $n_{f}=3$ and $\Lambda_{\mathrm{QCD}}^{(3)}=0.7 \mathrm{GeV}$. Dashed line (green in the web version): standard coupling $\alpha\left(Q^{2}\right)$; dotted line (blue in the web version): ghost-less or analytic coupling $\bar{\alpha}\left(Q^{2}\right)$; continuous line (red in the web version): cascade or time-like coupling $\tilde{\alpha}\left(k_{\perp}^{2}\right)$. 
Let us remark that an expansion in powers of the analytic coupling $\bar{\alpha}$ is an asymptotic expansion-as in the standard case-because the logarithmic structure of $\bar{\alpha}$ is the same as that of $\alpha$. As Fig. 2 clearly shows, the standard coupling and the ghost-less one are barely distinguishable at large scales [1].

\section{Effective coupling for gluon cascade}

As well known from perturbation theory, the emission of a gluon in a process is accompanied by an additional factor $\alpha$ in the cross-section, where $\alpha$ is the tree-level QCD coupling. In higher orders, one has to consider:

1. multiple emissions off the primary color charges - the heavy and the light quark in $B$ decays;

2. secondary emissions off the radiated gluons.

Primary multiple emissions produce the exponentiation of the one-gluon distribution while secondary emissions produce the decay of the radiated gluons into secondary partons-see Eq. (15). In the case of form factors, which are inclusive with respect to gluon decays, these higher-order terms have the main effect of replacing the tree-level coupling with an effective coupling evaluated at the transverse momentum of the primary emitted gluon [11]:

$$
\alpha \rightarrow \tilde{\alpha}\left(k_{\perp}^{2}\right)
$$

where

$$
\tilde{\alpha}\left(k_{\perp}^{2}\right) \equiv \frac{i}{2 \pi} \int_{0}^{k_{\perp}^{2}} d s \operatorname{Disc}_{s} \frac{\alpha(-s)}{s} .
$$

The coupling $\tilde{\alpha}\left(k_{\perp}^{2}\right)$ is characteristic of the QCD cascade and it is given by the integral of the discontinuity of the (interacting) gluon propagator over virtualities $s$ cut-off by the primary gluon transverse momentum. Let us remark that the cascade (or effective or time-like) coupling always refers to time-like kinematics.

The prescription at the root of our model is simply to replace the standard coupling on the r.h.s. of Eq. (37) with the ghost-less coupling constructed in the previous section:

$$
\tilde{\alpha}\left(k_{\perp}^{2}\right)=\frac{i}{2 \pi} \int_{0}^{k_{\perp}^{2}} d s \operatorname{Disc}_{s} \frac{\bar{\alpha}(-s)}{s} .
$$

If we neglect the $-i \pi$ terms in the integral over the discontinuity-i.e. the absorptive effectsthe cascade coupling exactly reduces to the ghost-less one:

$$
\tilde{\alpha}\left(k_{\perp}^{2}\right) \rightarrow \bar{\alpha}\left(k_{\perp}^{2}\right) \text {. }
$$

To render our model as accurate as possible, we include such absorptive effects and perform the integral on the r.h.s. of Eq. (38) exactly. By inserting the analytic coupling at LO in the integrand on the r.h.s. of Eq. (38), we obtain for the effective coupling:

$$
\tilde{\alpha}_{\mathrm{lo}}\left(k_{\perp}^{2}\right)=\frac{1}{2 \pi i \beta_{0}}\left[\log \left(\log \frac{k_{\perp}^{2}}{\Lambda^{2}}+i \pi\right)-\log \left(\log \frac{k_{\perp}^{2}}{\Lambda^{2}}-i \pi\right)\right] .
$$


At NLO, one has to add the contribution:

$$
\delta \tilde{\alpha}\left(k_{\perp}^{2}\right)=\frac{\beta_{1}}{2 \pi i \beta_{0}^{3}}\left[\frac{\log \left(\log k_{\perp}^{2} / \Lambda^{2}+i \pi\right)+1}{\log k_{\perp}^{2} / \Lambda^{2}+i \pi}-\frac{\log \left(\log k_{\perp}^{2} / \Lambda^{2}-i \pi\right)+1}{\log k_{\perp}^{2} / \Lambda^{2}-i \pi}\right] .
$$

The NNLO corrections read:

$$
\begin{aligned}
\delta \tilde{\alpha}^{\prime}\left(k_{\perp}^{2}\right)= & -\frac{\beta_{1}^{2}}{4 \pi i \beta_{0}^{5}}\left[\frac{\log ^{2}\left(\log k_{\perp}^{2} / \Lambda^{2}+i \pi\right)}{\left(\log k_{\perp}^{2} / \Lambda^{2}+i \pi\right)^{2}}-\frac{\log ^{2}\left(\log k_{\perp}^{2} / \Lambda^{2}-i \pi\right)}{\left(\log k_{\perp}^{2} / \Lambda^{2}-i \pi\right)^{2}}\right] \\
& +\frac{\beta_{1}^{2}-\beta_{0} \beta_{2}}{4 \pi i \beta_{0}^{5}}\left[\frac{1}{\left(\log k_{\perp}^{2} / \Lambda^{2}+i \pi\right)^{2}}-\frac{1}{\left(\log k_{\perp}^{2} / \Lambda^{2}-i \pi\right)^{2}}\right] .
\end{aligned}
$$

The time-like coupling in NNLO is simply the sum of the above terms:

$$
\tilde{\alpha}\left(k_{\perp}^{2}\right)=\tilde{\alpha}_{\mathrm{lo}}\left(k_{\perp}^{2}\right)+\delta \tilde{\alpha}\left(k_{\perp}^{2}\right)+\delta \tilde{\alpha}^{\prime}\left(k_{\perp}^{2}\right) .
$$

Let us make a few remarks:

1. the cascade coupling is very close to the ghost-less one for very small scales, let us say less than $1 \mathrm{GeV}$ (see Fig. 2). That is partly a consequence of the fact that both couplings have the same limit at zero momentum, $1 / \beta_{0}$, and is partly accidental [1]. The cascade coupling is instead smaller than the standard coupling or the ghost-less one in the perturbative region, at large scales, because it has an additional negative third-order contribution $\approx-1 / \log ^{3} Q^{2}-$ see next point;

2. the time-like coupling $\tilde{\alpha}$ has an expansion in powers of the standard $\overline{\mathrm{MS}}$ coupling $\alpha$ of the form:

$$
\tilde{\alpha}=\alpha-\frac{\left(\pi \beta_{0}\right)^{2}}{3} \alpha^{3}-\frac{5}{6} \frac{\beta_{1}}{\beta_{0}}\left(\pi \beta_{0}\right)^{2} \alpha^{4}+O\left(\alpha^{5}\right) .
$$

The relation above can be considered as an ordinary change of scheme for the coupling starting at third order;

3. the $\tilde{\beta}$ function for the time-like coupling, defined by the relation

$$
\frac{d \tilde{\alpha}}{d \log k_{\perp}^{2}}=\tilde{\beta}(\tilde{\alpha})=-\tilde{\beta}_{0} \tilde{\alpha}^{2}-\tilde{\beta}_{1} \tilde{\alpha}^{3}-\tilde{\beta}_{2} \tilde{\alpha}^{4}-\cdots,
$$

has a negative third-order coefficient, ${ }^{5}$

$$
\tilde{\beta}_{2}=\beta_{2}-\frac{1}{3}\left(\pi \beta_{0}\right)^{2} \beta_{0}<0,
$$

in agreement with the fact that the coupling saturates at small scales;

4. an expansion in powers of $\tilde{\alpha}\left(k_{\perp}^{2}\right)$ is not an asymptotic expansion for $k_{\perp}^{2} \rightarrow \infty$ because $\tilde{\alpha}$ even at LO contains infinitely many inverse powers of $\log k_{\perp}^{2}$.

The decoupling relations for the time-like coupling differ from the ones for the standard $\overline{\mathrm{MS}}$ coupling and read:

$$
\tilde{\alpha}_{n_{f}}=\tilde{\alpha}_{n_{f}-1}-\left(\frac{11}{72 \pi^{2}}-\frac{17}{54}+\frac{n_{f}}{54}\right) \tilde{\alpha}_{n_{f}-1}^{3},
$$

\footnotetext{
5 The first two coefficients are, as well known, invariant under a change of scheme: $\tilde{\beta}_{0}=\beta_{0}, \tilde{\beta}_{1}=\beta_{1}$.
} 
where $\tilde{\alpha}_{n_{f}}$ is the time-like coupling with $n_{f}$ active flavors and $\tilde{\alpha}_{n_{f}-1}$ with $n_{f}-1$. The above relation has to be imposed at a scale $\mu$ such that $\bar{m}(\mu)=\mu$, where $\bar{m}(\mu)$ is the $\overline{\mathrm{MS}}$ running mass of the decoupling quark.

Let us end this section summarizing the basic steps taken in the construction of the effective coupling for the gluon cascade of our model:

1. subtraction of the Landau pole from the ordinary QCD coupling;

2. inclusion of the absorptive effects related to the decay of time-like gluons in the coupling controlling jet evolution.

\subsection{Coupling in the DMW model}

Let us now evaluate the quantity

$$
\alpha_{0} \equiv \frac{1}{\mu_{I}} \int_{0}^{\mu_{I}} d k_{\perp} \tilde{\alpha}\left(k_{\perp}^{2}\right)
$$

parameterizing the leading non-perturbative effects in the Dokshitzer-Marchesini-Webber (DMW) model [5]. With $\mu_{I}=2 \mathrm{GeV}$ we obtain in our model $\alpha_{0}=0.40$ for $\alpha\left(m_{Z}\right)=0.12$ and $\alpha_{0}=0.44$ for $\alpha\left(m_{Z}\right)=0.125$. In general, we find that $\alpha_{0}$ is roughly linear in $\alpha\left(m_{Z}\right)$. A fit to $e^{+} e^{-}$shape variables data using next-to-leading resummed formulas gives $\alpha_{0} \simeq 0.45$. $^{6}$

\section{Threshold resummation with effective coupling}

In this section we describe a model for threshold resummation in semi-inclusive beauty decays based on the effective coupling considered in the previous section. Basically, we replace in the resummation exponent the standard coupling with the effective one.

\subsection{N-space}

In order to factorize multiple soft-gluon kinematic constraints, a transformation to $N$-space is required:

$$
\sigma_{N}(\alpha)=\int_{0}^{1}(1-t)^{N-1} \sigma(t ; \alpha) d t,
$$

where

$$
\sigma(t ; \alpha)=\delta(t)-\frac{C_{F} \alpha}{\pi}\left(\frac{\log t}{t}\right)_{+}-\frac{7 C_{F} \alpha}{4 \pi}\left(\frac{1}{t}\right)_{+}+O\left(\alpha^{2}\right),
$$

is the differential QCD form factor in the notation of [9]. $C_{F}=\left(N_{C}^{2}-1\right) /\left(2 N_{C}\right)=4 / 3$ with $N_{C}=3$ the number of colors and the plus distributions are defined as usual as:

$$
P(t)_{+} \equiv P(t)-\delta(t) \int_{0}^{1} P\left(t^{\prime}\right) d t^{\prime} .
$$

\footnotetext{
6 G. Salam: private communication.
} 
The form factor has an exponential form in $N$-space:

$$
\sigma_{N}(\alpha)=e^{G_{N}(\alpha)},
$$

where the exponent of the form factor reads:

$$
G_{N}(\alpha)=\int_{0}^{1} \frac{d y}{y}\left[(1-y)^{N-1}-1\right]\left\{\int_{Q^{2} y^{2}}^{Q^{2} y} \frac{d k_{\perp}^{2}}{k_{\perp}^{2}} \tilde{A}\left[\tilde{\alpha}\left(k_{\perp}^{2}\right)\right]+\tilde{B}\left[\tilde{\alpha}\left(Q^{2} y\right)\right]+\tilde{D}\left[\tilde{\alpha}\left(Q^{2} y^{2}\right)\right]\right\},
$$

with $Q=w m_{B}$ being the hard scale and $w \equiv 2 E_{X} / m_{B}$. The functions $\tilde{A}(\tilde{\alpha}), \tilde{B}(\tilde{\alpha})$ and $\tilde{D}(\tilde{\alpha})$ have expansions in powers of the effective coupling:

$$
\begin{aligned}
& \tilde{A}(\tilde{\alpha})=\sum_{n=1}^{\infty} \tilde{A}_{n} \tilde{\alpha}^{n}=\tilde{A}_{1} \tilde{\alpha}+\tilde{A}_{2} \tilde{\alpha}^{2}+\tilde{A}_{3} \tilde{\alpha}^{3}+\cdots ; \\
& \tilde{B}(\tilde{\alpha})=\sum_{n=1}^{\infty} \tilde{B}_{n} \tilde{\alpha}^{n}=\tilde{B}_{1} \tilde{\alpha}+\tilde{B}_{2} \tilde{\alpha}^{2}+\cdots \\
& \tilde{D}(\tilde{\alpha})=\sum_{n=1}^{\infty} \tilde{D}_{n} \tilde{\alpha}^{n}=\tilde{D}_{1} \tilde{\alpha}+\tilde{D}_{2} \tilde{\alpha}^{2}+\cdots
\end{aligned}
$$

The resummation constants for the cascade coupling are obtained from the standard ones (usually in the $\overline{\mathrm{MS}}$ scheme) by imposing equalities such as:

$$
\tilde{A}(\tilde{\alpha})=A(\alpha),
$$

where

$$
A(\alpha)=\sum_{n=1}^{\infty} A_{n} \alpha^{n}=A_{1} \alpha+A_{2} \alpha^{2}+A_{3} \alpha^{3}+\cdots
$$

is the standard double-logarithmic function. ${ }^{7}$ Expressing the cascade coupling in terms of the standard one, according to Eq. (44), we obtain ${ }^{8}$ :

$$
\begin{aligned}
& \tilde{A}_{1}=A_{1} ; \\
& \tilde{A}_{2}=A_{2} ; \\
& \tilde{A}_{3}=A_{3}+\frac{\left(\pi \beta_{0}\right)^{2}}{3} A_{1} ; \\
& \tilde{A}_{4}=A_{4}+\frac{2}{3}\left(\pi \beta_{0}\right)^{2} A_{2}+\frac{5}{6} \frac{\beta_{1}}{\beta_{0}}\left(\pi \beta_{0}\right)^{2} A_{1} .
\end{aligned}
$$

The first two coefficients $A_{1}$ and $A_{2}$ are the same for both couplings $\alpha$ and $\tilde{\alpha}$, while the thirdorder one $A_{3}$ is modified going to the time-like coupling by a contribution proportional to the

\footnotetext{
7 A compilation of the resummation constants in our normalization, with references to the original papers, can be found in [9].

8 Analogous relations hold for the $\tilde{B}_{i}$ 's and the $\tilde{D}_{i}$ 's.
} 
first-order coefficient. For $n_{f}=3, \tilde{A}_{3} \approx 1$ is larger than $A_{3} \approx 0.3$ by a factor 3 , but it is still acceptably small.

We have found that the inclusion of the NNLO terms involving $\tilde{A}_{3}, B_{2}$ and $D_{2}$-in particular $\tilde{A}_{3}$ - is crucial for a good description of the experimental data. The NLO spectra are indeed peaked at too low hadron invariant masses and a sizable and positive value for $\tilde{A}_{3}$ suppresses the elastic region and shifts the spectra to higher $m_{X}$ 's. The model could be improved by including NNNLO terms, which require the knowledge of the coefficients $A_{4}, B_{3}$ and $D_{3}$; at present, only $B_{3}$ is analytically known [12].

Our model has been constructed by means of a power expansion in a single (effective) coupling $\tilde{\alpha}$, i.e. higher orders are proportional to $\tilde{\alpha}^{n}$. In [1] and in [13] a non-power expansion had been proposed involving a different coupling for any $n$, which has interesting theoretical properties. In second order $(n=2)$, for example, one has the coupling

$$
\widetilde{\alpha^{2}}\left(k_{\perp}^{2}\right)=\frac{1}{\beta_{0}^{2}\left(\pi^{2}+\log ^{2} k_{\perp}^{2} / \Lambda^{2}\right)}
$$

in place of $\tilde{\alpha}\left(k_{\perp}^{2}\right)^{2}$, with $\tilde{\alpha}\left(k_{\perp}^{2}\right)$ given by Eq. (40). We have found that the non-power expansion does not offer a good description of the measured spectra. That is because

$$
\widetilde{\alpha^{2}}\left(k_{\perp}^{2}\right) \rightarrow 0 \quad \text { while } \tilde{\alpha}\left(k_{\perp}^{2}\right)^{2} \rightarrow \frac{1}{\beta_{0}^{2}} \approx O(1) \text { for } k_{\perp}^{2} \rightarrow 0 .
$$

That implies that second-order effects are suppressed in the soft region with the non-power expansion compared to the power expansion case. In general, the non-power expansion renders the higher-order effects very small [1]. But, as discussed above, in beauty decays, sizable third-order effects are needed to take the theoretical curves close to the data, disfavoring the non-power expansion.

In order to include as many corrections as possible-higher order $\log N$ terms, $1 / N$ contributions, etc.-in agreement with the philosophy described in the introduction, we make the integration over $y$ in $G_{N}$ exactly, in numerical way. This is possible because the time-like coupling $\tilde{\alpha}\left(k_{\perp}^{2}\right)$, unlike the standard one, does not have the Landau singularity and is regular for any $k_{\perp}^{2} \geqslant 0$.

\subsection{Inverse transform}

The form factor in momentum space is obtained by inverse transform:

$$
\sigma(t ; \alpha)=\int_{C-i \infty}^{C+i \infty} \frac{d N}{2 \pi i}(1-t)^{-N} \sigma_{N}(\alpha)
$$

where the constant $C$ is chosen so that the integration contour in the $N$-plane lies to the right of all the singularities of $\sigma_{N}(\alpha)$. In order to correctly implement multi-parton kinematics, the inverse transform from $N$-space back to $x$-space is also made exactly in numerical way. Let us note that no prescription — such as the minimal prescription in the standard formalism [4] -is needed in our model because $\sigma_{N}(\alpha)$ is analytic for $\operatorname{Re} N>0$. 


\section{Radiative decay}

The event fraction or partially-integrated rate for the radiative $B$ decay

$$
B \rightarrow X_{s}+\gamma
$$

can be written as $[14,15]$ :

$$
\frac{1}{\Gamma_{r}^{(0)}} \int_{0}^{t} \frac{d \Gamma_{r}}{d t^{\prime}} d t^{\prime}=K_{r}(\alpha) \Sigma(t ; \alpha)+D_{r}(t ; \alpha)
$$

where

$$
t \equiv \frac{m_{X}^{2}}{m_{B}^{2}}
$$

is a dimensionless variable,

$$
\Sigma(t ; \alpha)=\int_{0}^{t} \sigma\left(t^{\prime} ; \alpha\right) d t^{\prime}=1-\frac{C_{F} \alpha}{2 \pi} \log ^{2} t-\frac{7 C_{F} \alpha}{4 \pi} \log t+O\left(\alpha^{2}\right)
$$

is the partially-integrated form factor and

$$
\Gamma_{r}^{(0)}=\frac{\alpha_{\mathrm{em}}}{\pi} \frac{G_{F}^{2}\left|V_{t b} V_{t s}^{\star}\right|^{2} m_{b}^{3} \bar{m}_{b}^{2}}{32 \pi^{3}} C_{7}^{2}
$$

is the lowest-order inclusive width. $m_{b} \approx 5 \mathrm{GeV}$ is the beauty pole mass while $\bar{m}_{b}$ is the $\overline{\mathrm{MS}}$ mass evaluated in $\mu=m_{b}$. Their relation reads:

$$
\bar{m}_{b}=\left[1-\frac{\alpha\left(m_{b}\right) C_{F}}{\pi}+O\left(\alpha^{2}\right)\right] m_{b} \simeq 0.9 m_{b}
$$

$K_{r}(\alpha)$ is a short-distance coefficient function specific for this process and having an expansion in powers of $\alpha$ :

$$
K_{r}(\alpha)=1+\alpha K_{r}^{(1)}+\alpha^{2} K_{r}^{(2)}+O\left(\alpha^{3}\right) .
$$

The explicit expression of the first-order term reads:

$$
K_{r}^{(1)}=\frac{1}{2 \pi} \sum_{i=1}^{8} \frac{C_{i}}{C_{7}} \operatorname{Re} r_{i}
$$

where the $C_{i}$ 's are short-distance coefficient functions entering the effective $b \rightarrow s \gamma$ Hamiltonian, $\mathcal{H}^{b \rightarrow s \gamma}$, whose numerical values are given in the appendix, and the $r_{i}$ 's are complex constants. $D_{r}(t ; \alpha)$ is a process-dependent remainder function, which is included to correctly describe also the high jet mass region $t \approx O(1)$. In our leading-twist analysis, this function can be computed in perturbation theory and starts in first order:

$$
D_{r}(t ; \alpha)=\alpha D_{r}^{(1)}(t)+\alpha^{2} D_{r}^{(2)}(t)+O\left(\alpha^{3}\right),
$$

with

$$
D_{r}^{(1)}(t)=\frac{1}{\pi} \sum_{i \leqslant j}^{1,8} \frac{C_{i} C_{j}}{C_{7}^{2}} f_{i j}^{(1)}(t) .
$$


The $f_{i j}(t)$ 's are functions whose explicit expressions are given in the appendix.

Since the total width $\Gamma_{r}$ is infrared divergent beyond tree level, because of soft photon effects occurring in the spectrum for $t \rightarrow 1$, it is convenient to define an event fraction normalized to the partial rate

$$
\Gamma_{r}(\delta) \equiv \int_{0}^{\delta} \frac{d \Gamma_{r}}{d t^{\prime}} d t^{\prime},
$$

where $\delta<1$ is a parameter. That is also convenient for experimental reasons: due to large backgrounds, the presently accessible range of hadron masses is at the most $0<t<0.3$ (see later). The event fraction normalized to $\Gamma_{r}(\delta)$ reads:

$$
R_{\delta}(t)=\frac{1}{\Gamma_{r}(\delta)} \int_{0}^{t} \frac{d \Gamma_{r}}{d t^{\prime}} d t^{\prime} .
$$

For $\delta \rightarrow 1, R_{\delta}(t)$ tends to the standard event fraction $R_{r}(t)$. The normalization condition is:

$$
R_{\delta}(\delta)=1 .
$$

The differential spectrum is obtained by differentiation:

$$
\frac{d \Gamma_{r}}{d t}=\Gamma_{r}(\delta) \frac{d R_{\delta}}{d t} .
$$

A resummed expression of the following form holds:

$$
R_{\delta}(t ; \alpha)=C_{\delta}(\alpha) \Sigma_{\delta}(t ; \alpha)+D_{\delta}(t ; \alpha),
$$

where we have defined the form factor

$$
\Sigma_{\delta}(t ; \alpha) \equiv \frac{\Sigma(t ; \alpha)}{\Sigma(\delta ; \alpha)},
$$

which is normalized as

$$
\Sigma_{\delta}(\delta ; \alpha)=1 .
$$

The normalization condition (78) gives:

$$
C_{\delta}(\alpha)+D_{\delta}(\delta ; \alpha)=1 .
$$

The expansions of the coefficient function and the remainder function read:

$$
\begin{aligned}
& C_{\delta}(\alpha)=1+\alpha C_{\delta}^{(1)}+\alpha^{2} C_{\delta}^{(2)}+O\left(\alpha^{3}\right) ; \\
& D_{\delta}(t ; \alpha)=\alpha D_{\delta}^{(1)}(t)+\alpha^{2} D_{\delta}^{(2)}(t)+O\left(\alpha^{3}\right),
\end{aligned}
$$

with

$$
C_{\delta}^{(1)}=-\frac{D_{r}^{(1)}(\delta)}{\Sigma(\delta ; \alpha)}, \quad D_{\delta}^{(1)}(t)=\frac{D_{r}^{(1)}(t)}{\Sigma(\delta ; \alpha)} .
$$

Note that we only expand $D_{r}(t ; \alpha)$ in powers of $\alpha$ and not $\Sigma(\delta ; \alpha)$, because, for sufficient small $\delta$, one can have $\alpha \log ^{2} \delta \approx O(1)$, implying need for resummation to any order in $\alpha$. For $\delta=0.26$, one obtains $C_{\delta}^{(1)} \simeq-0.48$, i.e. a $O(10 \%)$ correction to the tree-level coefficient function $(\Sigma(t=$ $0.26 ; \alpha)=1.10)$. 


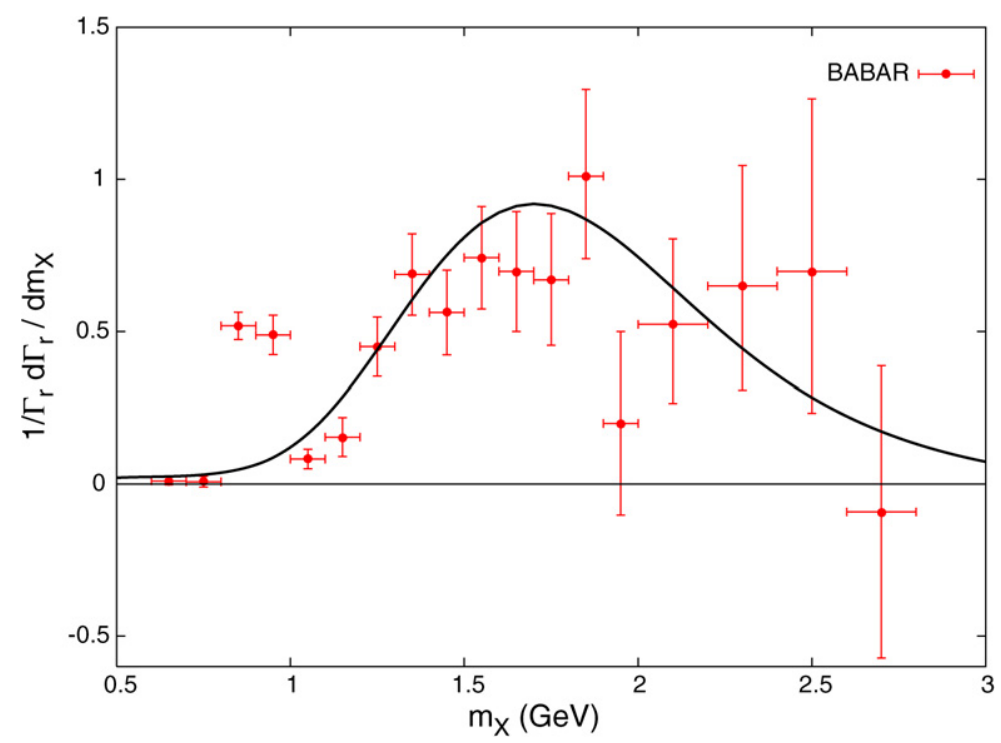

Fig. 3. $B \rightarrow X_{S} \gamma$ invariant hadron mass distribution from BaBar compared to our model for $\alpha_{S}\left(m_{Z}\right)=0.123$. The theory and the data are normalized to one in the experimentally accessible region.

\subsection{Phenomenology}

In Fig. 3 we compare the invariant hadron mass distribution for the radiative decay, $d \Gamma_{r} / d m_{X}$, obtained with our model with experimental data from the BaBar Collaboration [16]. The data show a rather pronounced $K^{*}$ peak, which clearly cannot be accounted for in a perturbative QCD framework. ${ }^{9}$ We have therefore discarded in the analysis the data points with $m_{X}<1.1 \mathrm{GeV} .{ }^{10}$ We obtain a minimum $\chi^{2}=12$ for $\alpha_{S}\left(m_{Z}\right)=0.1255$ for 13 data points, i.e. for 12 degrees of freedom (d.o.f.) because of the fixed normalization. Since we deal with the standard QCD coupling, let us write $\alpha_{S}$ from this section till the end of the paper. To improve the agreement of the theory with the data and to estimate the error on $\alpha_{S}\left(m_{Z}\right)$, we have performed a Gaussian smearing of $\Delta m_{X}=300 \mathrm{MeV}$ of the data points and of the theoretical distribution, and we have discarded the points with $m_{X}<800 \mathrm{MeV}$. We obtain a minimum $\chi^{2}=6.8$ for $\alpha_{S}\left(m_{Z}\right)=0.1205$ for 15 d.o.f. By taking as an estimate of $\alpha_{S}\left(m_{Z}\right)$ the average of the above values and as an estimate of the error their difference, we quote ${ }^{11}$ :

$$
\alpha_{S}\left(m_{Z}\right)=0.123 \pm 0.003 \quad\left(m_{X s}: \quad \text { BaBar }\right)
$$

\footnotetext{
9 To have a point-to-point description of the data, one has to include by hand the contribution of this resonance (without modifying the total rate), by means of one or more free parameters.

$10 m_{K^{*}}=892 \mathrm{MeV}$ and $\Gamma_{K^{*}}=51 \mathrm{MeV}$.

11 We have taken $\bar{m}_{b}=4.8 \mathrm{GeV}, \bar{m}_{c}=1.4 \mathrm{GeV}$ and $\bar{m}_{s}=0.3 \mathrm{GeV}$ in the decoupling relations. In general, changing the $\overline{\mathrm{MS}}$ masses in a reasonable range modifies the theoretical predictions in a negligible way. Increasing the $\overline{\mathrm{MS}}$ masses is roughly equivalent to a slight increase of $\alpha_{S}\left(m_{Z}\right)$. That is because, lowering the renormalization scale, the QCD coupling rises faster for a smaller number of active flavors.
} 


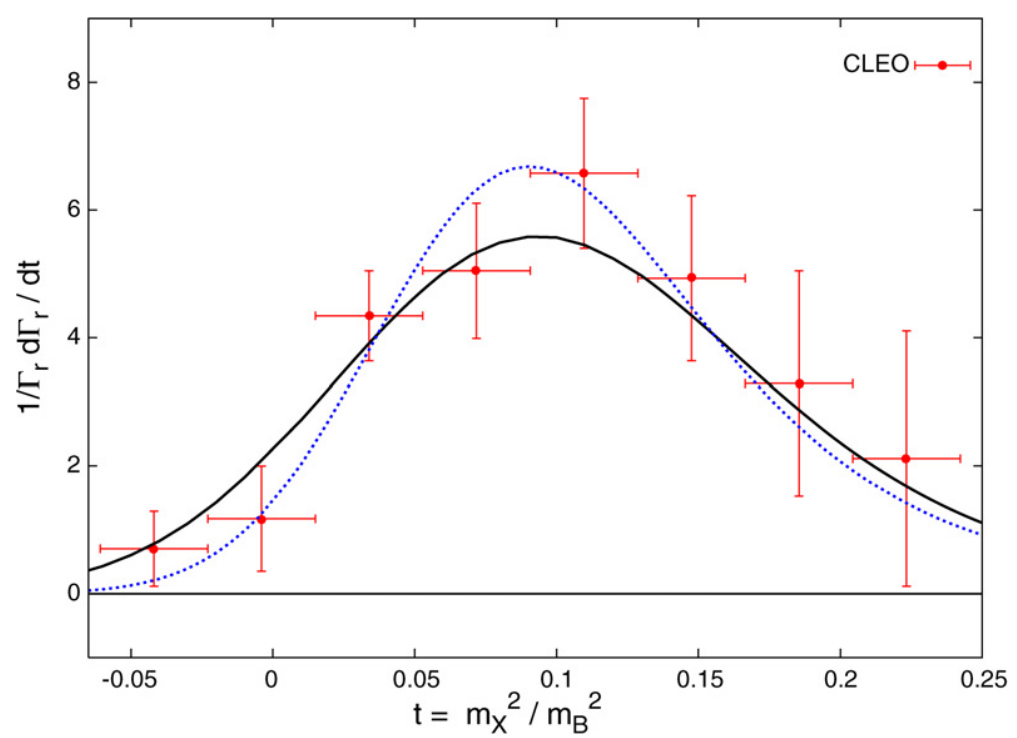

Fig. 4. $B \rightarrow X_{S} \gamma$ photon spectrum from CLEO compared to our model. Dotted line (blue in the web version): $\alpha_{S}\left(m_{Z}\right)=0.118$ and $\sigma_{\gamma}=100 \mathrm{MeV}$ to model the Doppler effect (see text); continuous line (black): $\alpha_{S}\left(m_{Z}\right)=0.117$ and $\sigma_{\gamma}=150 \mathrm{MeV}$.

In Fig. 4 we compare the photon energy spectrum computed in the framework of our model with data from the CLEO Collaboration [17]. In the $B$ rest-frame,

$$
t=1-\frac{2 E_{\gamma}}{m_{B}}
$$

The photon energies are however measured in the $\Upsilon(4 S)$ rest frame, in which the $B$ mesons have a small, non-relativistic motion. In order to model the Doppler effect, we have convoluted the theoretical curve for $E_{\gamma}$-computed with a $B$ meson at rest-with a normal distribution of $\sigma_{\gamma}=150 \mathrm{MeV}$, as suggested by CLEO itself. Let us note that the Doppler effect is sufficient to completely wash out the $K^{*}$ peak. We obtain a minimum $\chi^{2}=3.8$ for $\alpha_{S}\left(m_{Z}\right)=0.117$ for 7 d.o.f. Assuming complete independence of the experimental points, we allow the $\chi^{2}$ to raise by one unit to estimate the error and we obtain:

$$
\alpha_{S}\left(m_{Z}\right)=0.117 \pm 0.004 \quad\left(E_{\gamma}: \quad \text { CLEO }, \sigma_{\gamma}=150 \mathrm{MeV}\right) .
$$

To check the modeling above of the Doppler effect, we have used the following method. We have converted the $m_{X s}$ distribution by BaBar above to a photon spectrum in the $B$ rest-frame and we have convoluted it with a normal distribution with a variable $\sigma_{\gamma}$, obtaining the points $\left(x_{i}, y_{i}^{\prime}\left(\sigma_{\gamma}\right), \sigma_{i}^{\prime}\right)$. We have then minimized the quantity

$$
H\left(\sigma_{\gamma}\right) \equiv \sum_{i} \frac{\left[y_{i}-y_{i}^{\prime}\left(\sigma_{\gamma}\right)\right]^{2}}{\sigma_{i}^{2}+\sigma_{i}^{\prime 2}}
$$




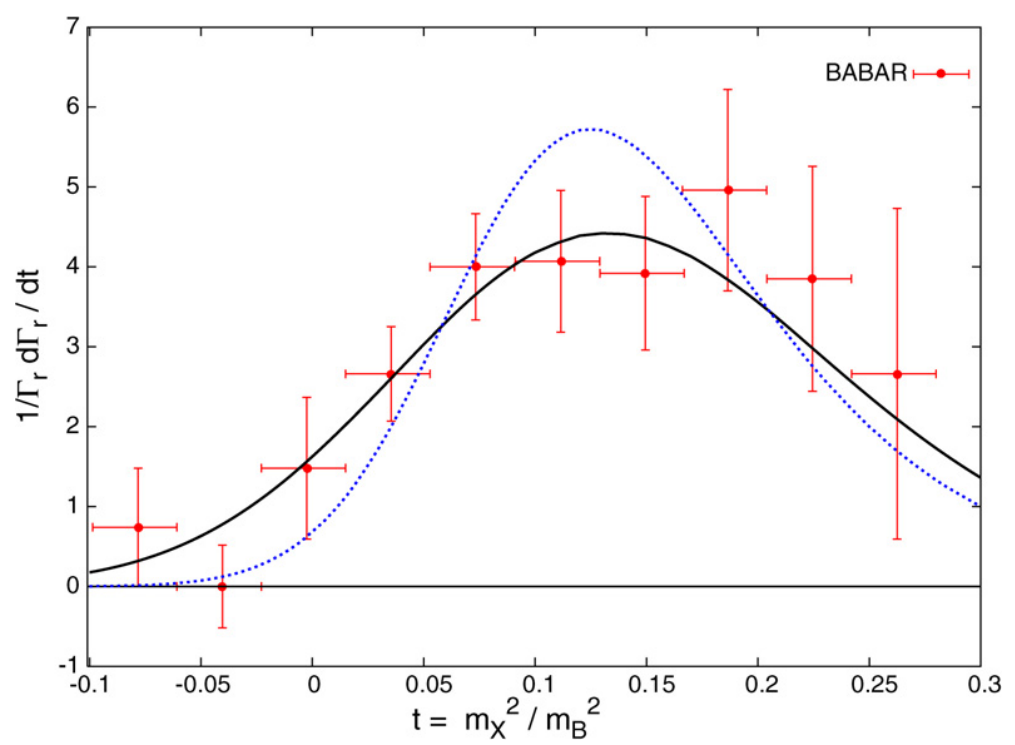

Fig. 5. $B \rightarrow X_{S} \gamma$ photon spectrum from BaBar compared to our model. Dotted line (blue in the web version): $\alpha_{S}\left(m_{Z}\right)=0.130$ and $\sigma_{\gamma}=100 \mathrm{MeV}$; continuous line (black): $\alpha_{S}\left(m_{Z}\right)=0.129$ and $\sigma_{\gamma}=200 \mathrm{MeV}$.

with respect to $\sigma_{\gamma}$, where $\left(x_{i}, y_{i}, \sigma_{i}\right)$ are the CLEO data. ${ }^{12}$ We have found a minimum of $H\left(\sigma_{\gamma}\right)$ for $\sigma_{\gamma} \simeq 100 \mathrm{MeV}$, which gives similar results to the analysis with $\sigma_{\gamma}=150 \mathrm{MeV}$ :

$$
\alpha_{S}\left(m_{Z}\right)=0.118 \pm 0.003 \quad\left(E_{\gamma}: \quad \text { CLEO, } \sigma_{\gamma}=100 \mathrm{MeV}\right),
$$

with $\chi^{2}=3.4$. As intuitively expected, reducing $\sigma_{\gamma}$ produces a shaper spectrum.

In Fig. 5 we compare the predictions of our model with a spectrum from the BaBar Collaboration [18]. The BaBar spectrum is somewhat softer than the CLEO one-even though the difference is within one standard deviation; we have interpreted this difference as a resolution effect and we have convoluted our theoretical curve with a normal distribution with a slightly larger standard deviation, $\sigma_{\gamma}=200 \mathrm{MeV} .{ }^{13}$ We obtain a minimum $\chi^{2}=5.1$ for 9 d.o.f. Performing a similar analysis as for the CLEO data, we obtain:

$$
\alpha_{S}\left(m_{Z}\right)=0.129 \pm 0.005 \quad\left(E_{\gamma}: \quad \text { BaBar, } \sigma_{\gamma}=200 \mathrm{MeV}\right) .
$$

Following the minimization procedure above for the CLEO spectrum (see Eq. (90)), we obtain also for the BaBar photon spectrum $\sigma_{\gamma} \simeq 100 \mathrm{MeV}$, to give:

$$
\alpha_{S}\left(m_{Z}\right)=0.130 \pm 0.008 \quad\left(E_{\gamma}: \quad \text { BaBar, } \sigma_{\gamma}=100 \mathrm{MeV}\right),
$$

with $\chi^{2}=8$.0. Let us note that $\sigma_{\gamma}$ and $\alpha_{S}\left(m_{Z}\right)$ are slightly anti-correlated because by increasing $\alpha_{S}\left(m_{Z}\right)$ more radiation is emitted with a smearing effect similar to the one of increasing $\sigma_{\gamma}$.

The same analysis on the BaBar photon spectrum can be repeated for the Belle one [19] (see Fig. 6). The minimization of $H\left(\sigma_{\gamma}\right)$ gives in this case $\sigma_{\gamma} \simeq 200 \mathrm{MeV}$. We obtain a minimum of

\footnotetext{
12 Let us remark however that the two spectra entering Eq. (90) are independent on each other.

13 A more sophisticated analysis from the experimentalists, including the true resolution functions, is strongly encouraged!
} 


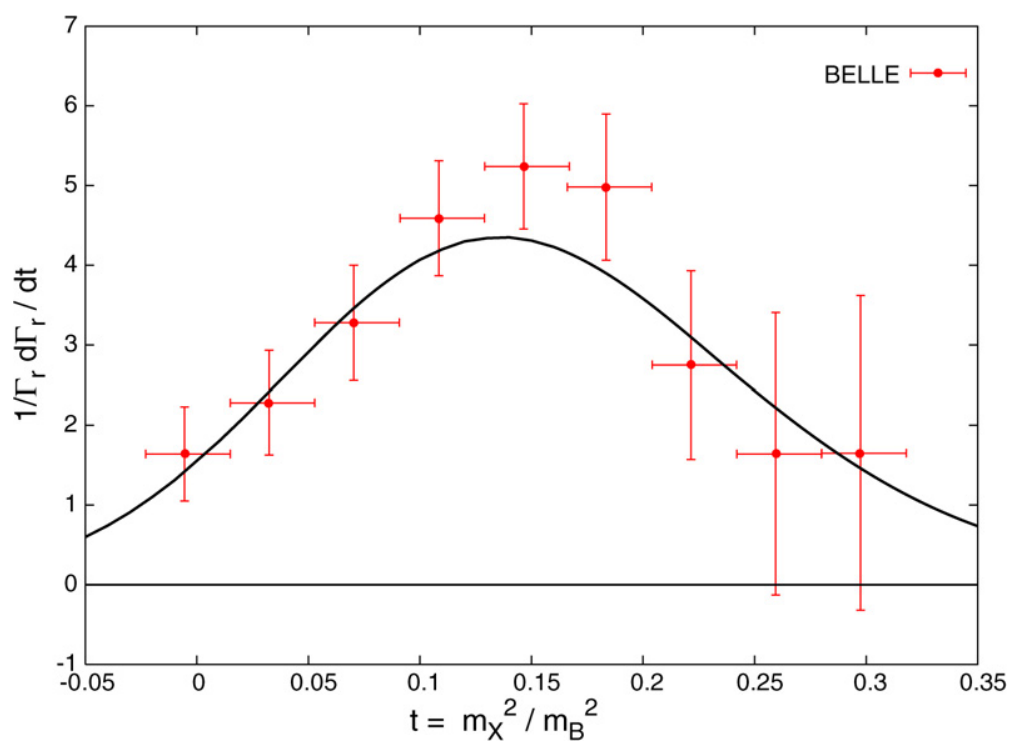

Fig. 6. $B \rightarrow X_{S} \gamma$ photon spectrum from Belle compared to our model for $\alpha_{S}\left(m_{Z}\right)=0.130$ and $\sigma_{\gamma}=200 \mathrm{MeV}$.

$\chi^{2}=5.3$ for 8 d.o.f., to give

$$
\alpha_{S}\left(m_{Z}\right)=0.130 \pm 0.005 \quad\left(E_{\gamma}: \text { Belle, } \sigma_{\gamma}=200 \mathrm{MeV}\right) .
$$

The over-all picture is that there is a good agreement of our model with the data in the region $m_{X}>1 \mathrm{GeV}$, below which single resonances such as $K$ and $K^{*}$ are expected to have a substantial effect in the dynamics. The extracted values of $\alpha_{S}\left(m_{Z}\right)$ are in agreement with the world average [30]

$$
\alpha_{S}\left(m_{Z}\right)=0.1176 \pm 0.0020
$$

at most within two standard deviations.

\section{Semileptonic decay}

Resummed expressions for the triple-differential distribution in the inclusive charmless semileptonic $B$ decays,

$$
B \rightarrow X_{u}+l+v,
$$

as well as for many double and single distributions have been given in [9,14,20,21], so we do not repeat them here. ${ }^{14}$ To compare with semileptonic data, we just supplement these resummed expressions with the QCD form factor $\sigma$ computed within our model.

14 A slightly different formalism, which uses light-cone variables and is equivalent to ours in leading twist, has been developed in [22]. 


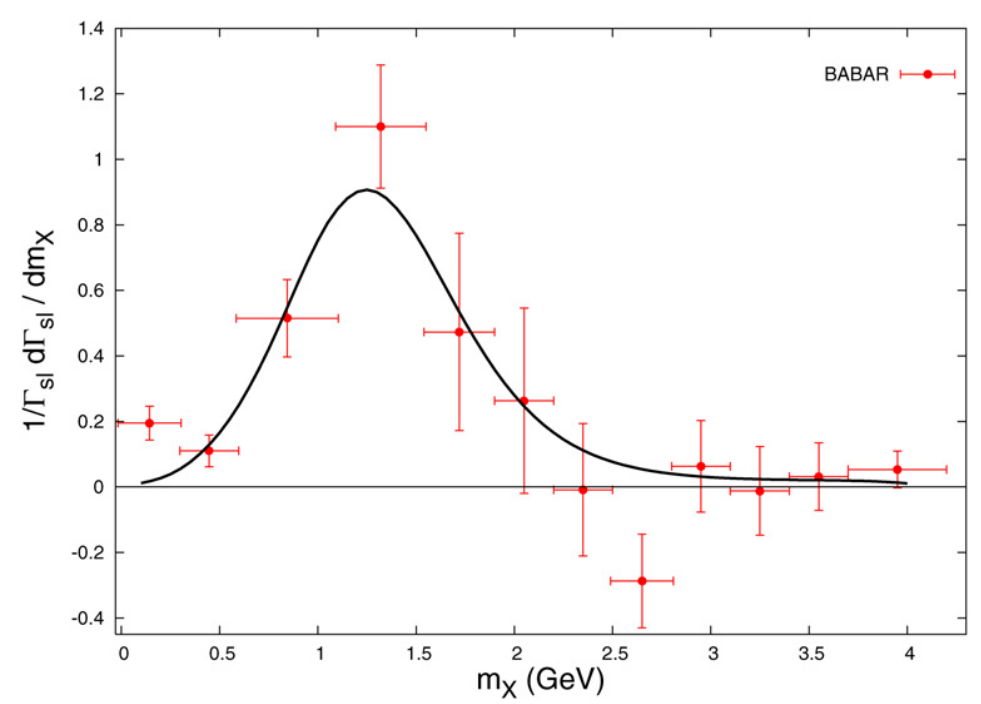

Fig. 7. Invariant hadron mass distribution in semileptonic decays from BaBar compared to our model for $\alpha_{S}\left(m_{Z}\right)=0.119$.

\subsection{Hadron mass distribution}

In Fig. 7 we compare the invariant hadron mass distribution in the semileptonic decay (96) in our model with data from the BaBar Collaboration [23]. We discard the point with $m_{X}<$ $400 \mathrm{MeV}$, which is dominated by the $\pi$ peak, and the points with $m_{X}>2.6 \mathrm{GeV}$, which give basically no information on the signal. ${ }^{15}$ We obtain a minimum $\chi^{2}=1.1$ for 5 d.o.f. and, using the method of the previous section, we obtain:

$$
\alpha_{S}\left(m_{Z}\right)=0.119 \pm 0.003 \quad\left(m_{X u}: \quad \text { BaBar }\right)
$$

Since the $\rho$ width is larger than that of the $K^{*}, \Gamma_{\rho} \simeq 150 \mathrm{MeV} \simeq 3 \Gamma_{K^{*}}$, and the binning is rather large $\left(\Delta m_{X}=400 \mathrm{MeV}\right)$, we do not apply any smearing procedure in this case.

In Fig. 8 we present a similar plot with Belle data [24]. To extract $\alpha_{S}\left(m_{Z}\right)$, we discard the first 7 points, having $m_{X}<0.8 \mathrm{GeV}$. We obtain a minimum $\chi^{2}=5.3$ with 7 d.o.f., to give $\alpha_{S}\left(m_{Z}\right)=$ $0.123 \pm 0.006$. Since the binning is smaller for Belle $\left(\Delta m_{X}=120 \mathrm{MeV}\right)$ than for BaBar, the $\rho$ peak is pretty visible now. To reduce the resonance effect, we convolve our theoretical curve and the experimental data with a normal distribution of $\sigma=300 \mathrm{MeV}$, as we have made with the $m_{X s}$ spectrum in the previous section. By discarding the first four points, we obtain a minimum $\chi^{2}=0.41$ for 10 d.o.f. to give $\alpha_{S}\left(m_{Z}\right)=0.115 \pm 0.004$. Combining the above measures as we have made for the $m_{X s}$ spectrum, we quote:

$$
\alpha_{S}\left(m_{Z}\right)=0.119 \pm 0.004 \quad\left(m_{X u}: \quad \text { Belle }\right)
$$

$\overline{15}$ Let us stress that for $m_{X}>1.7 \mathrm{GeV}$ experimental errors become very large because of the large background coming from semileptonic $b \rightarrow c$ transitions which have $m_{X} \geqslant m_{D}=1.867 \mathrm{GeV}$. 


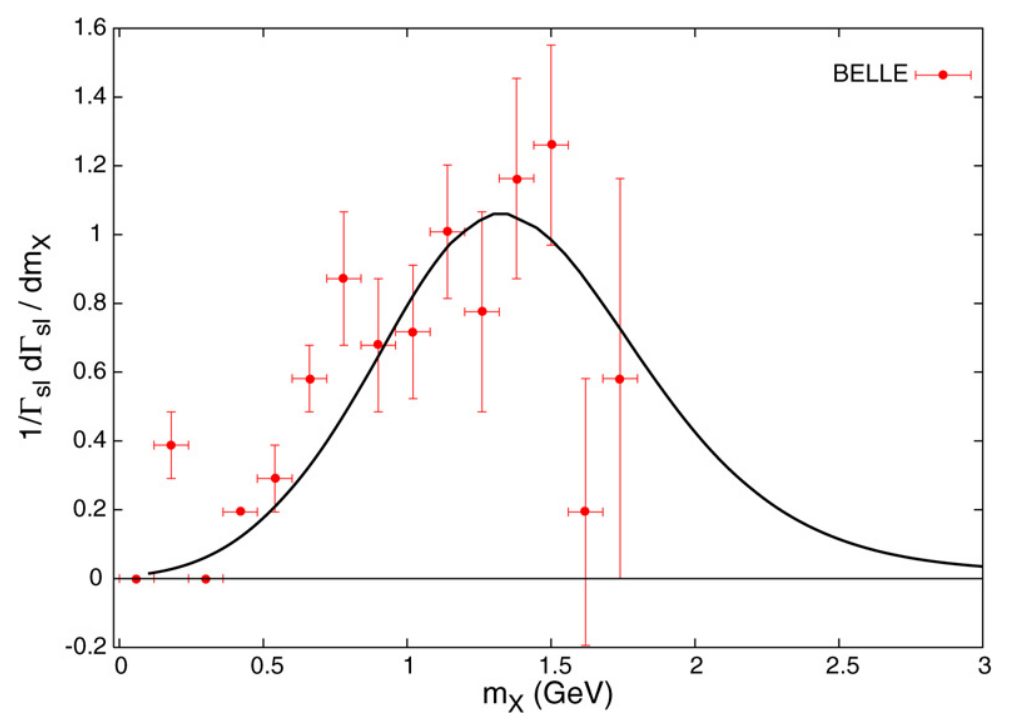

Fig. 8. Invariant hadron mass distribution in semileptonic decay from Belle compared to our model for $\alpha_{S}\left(m_{Z}\right)=0.123$.

Let us note that semileptonic distributions peak at smaller hadron masses than radiative ones because they have a smaller average hadron energy [9]:

$$
\left\langle E_{X}\right\rangle_{\mathrm{sl}} \simeq 0.7\left\langle E_{X}\right\rangle_{\mathrm{rd}}
$$

For $\alpha_{S}\left(m_{Z}\right)=0.123$, we find for the peak positions in our model:

$$
m_{X u} \approx 1.3 \mathrm{GeV} \quad \text { while } m_{X s} \approx 1.7 \mathrm{GeV} .
$$

We end this section by noting that the extracted values of $\alpha_{S}\left(m_{Z}\right)$ from the above measurements are in agreement with each other as well with the reference value within one standard deviation.

\subsection{Electron spectrum}

The electron spectrum in the decay (96) is affected by a large background for

$$
E_{e}<\frac{m_{B}}{2}\left(1-\frac{m_{D}^{2}}{m_{B}^{2}}\right) \simeq 2.31 \mathrm{GeV}
$$

coming from the decays

$$
B \rightarrow X_{c}+l+v .
$$

This background is larger than the signal by two orders of magnitude because $\left|V_{u b}\right|^{2} /\left|V_{c b}\right|^{2} \sim$ $10^{-2}$. In order to avoid the large errors coming from its subtraction, we have normalized the theory and the data to one in the region $E_{e}>2.31 \mathrm{GeV}$. Instead of the electron energy, we prefer to use the variable

$$
\bar{x}_{e} \equiv 1-\frac{2 E_{e}}{m_{B}},
$$




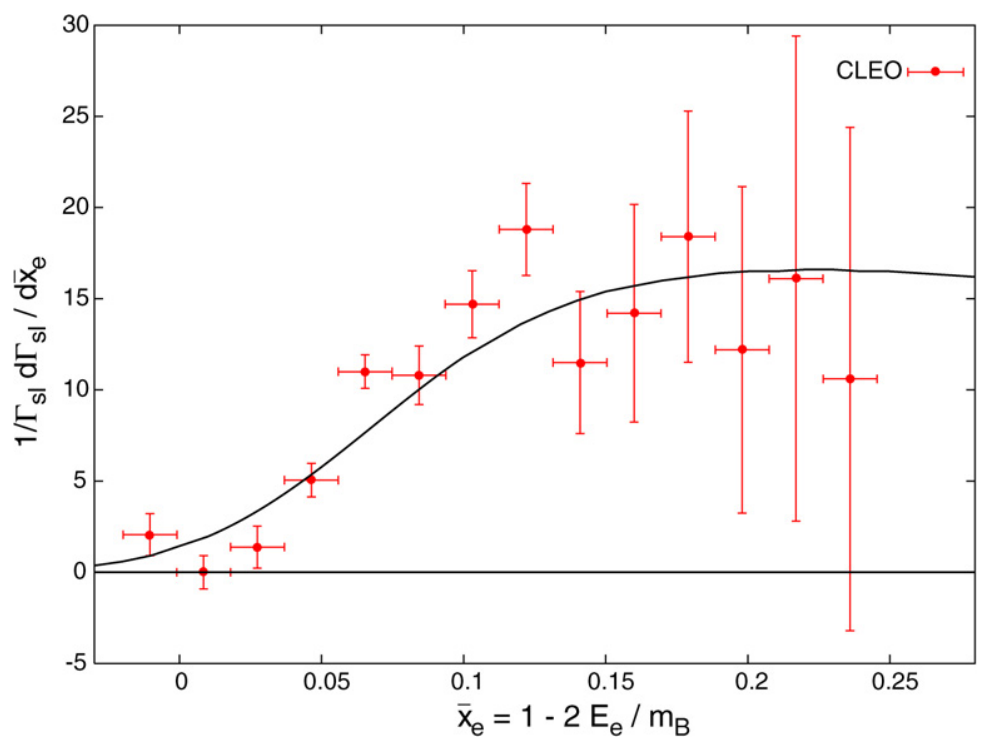

Fig. 9. Electron spectrum in semileptonic charmless $B$ decay from CLEO compared to our model with $\alpha_{S}\left(m_{Z}\right)=0.118$. The data and the theory are normalized to one in the charm background free region $0<\bar{x}_{e}<0.125$.

which is equal to zero for the largest electron energy. The charm background occurs for $\bar{x}_{e}>$ 0.125. To include the Doppler effect, we convolve our spectra with a normal distribution of standard deviation $\sigma_{e}=100 \mathrm{MeV}$.

In Fig. 9 we compare the electron spectrum in our model ${ }^{16}$ with data from the CLEO Collaboration [25]. We obtain a minimum $\chi^{2}=30$ for 13 d.o.f. and with the analysis described above we obtain:

$$
\alpha_{S}\left(m_{Z}\right)=0.117 \pm 0.005 \quad\left(E_{e}: \quad \text { CLEO }\right) .
$$

The over-all agreement of the model with the data is acceptable in all the measured range of electron energies. In the region affected by the charm background, experimental errors become however very large.

In Fig. 10 we compare our prediction with the electron spectrum measured by the BaBar Collaboration [26]. In the $\chi^{2}$ analysis we remove the 4 points with the smallest electron energies, which are affected by the subtraction of the charm background. We obtain a minimum $\chi^{2}=16$ for 9 d.o.f. and we obtain

$$
\alpha_{S}\left(m_{Z}\right)=0.119 \pm 0.005 \quad\left(E_{e}: \text { BaBar }\right) .
$$

In Fig. 11 we compare our model with Belle data [27]. For the $\chi^{2}$ analysis we have discarded the seven points with the largest $\bar{x}_{e}$, i.e. with smallest electron energies. We obtain a minimum $\chi^{2}=7$ for 8 d.o.f. for $\alpha_{S}\left(m_{Z}\right) \approx 0.135$. Since the $\chi^{2}$ is a rather irregular function of $\alpha_{S}\left(m_{Z}\right)$ in this case—without a well-shaped minimum—we are not able to estimate the error.

\footnotetext{
16 Let us note that the tree-level electron spectrum has a maximum for $\bar{x}_{e}=0$, at the largest electron energy, where it is flat. The shift of the maximum inside the kinematical domain, in $\bar{x}_{e} \approx 0.2$, is a Sudakov effect. Because of infrared divergencies, soft radiation is always emitted and the high energy electron recoils against a neutrino and a massive up-quark jet, instead of a massless one.
} 


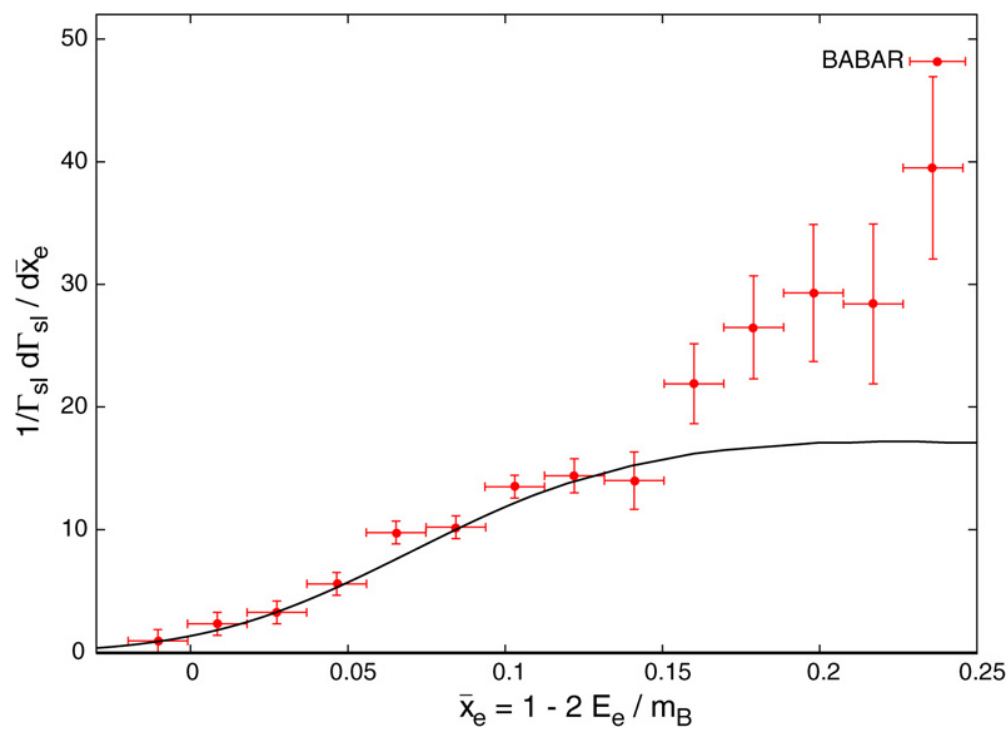

Fig. 10. Electron spectrum in semileptonic decay from BaBar compared to our model with $\alpha_{S}\left(m_{Z}\right)=0.119$. The data and the theory are normalized to one in the charm background free region $0<\bar{x}_{e}<0.125$.

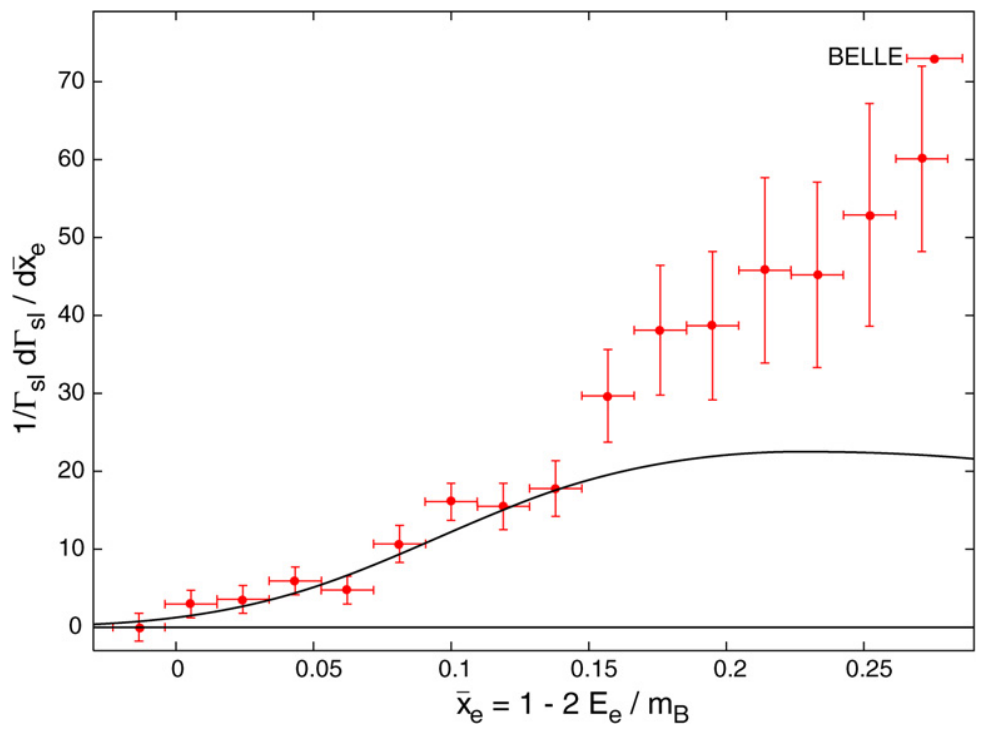

Fig. 11. Electron spectrum in semileptonic decay from Belle compared to our model for $\alpha_{S}\left(m_{Z}\right)=0.135$. The data and the theory are normalized to one in the charm background free region $0<\bar{x}_{e}<0.125$.

Finally, in Fig. 12 we compare our model for $\alpha_{S}\left(m_{Z}\right)=0.119$ with a preliminary measure of the electron spectrum of the BaBar Collaboration extending down to $E_{e}=1.1 \mathrm{GeV}$ [28]. As it is clearly seen, the theoretical spectrum is harder than the experimental one. We do not known whether this discrepancy is related to a deficiency of our model or to an under-estimated charm background. 


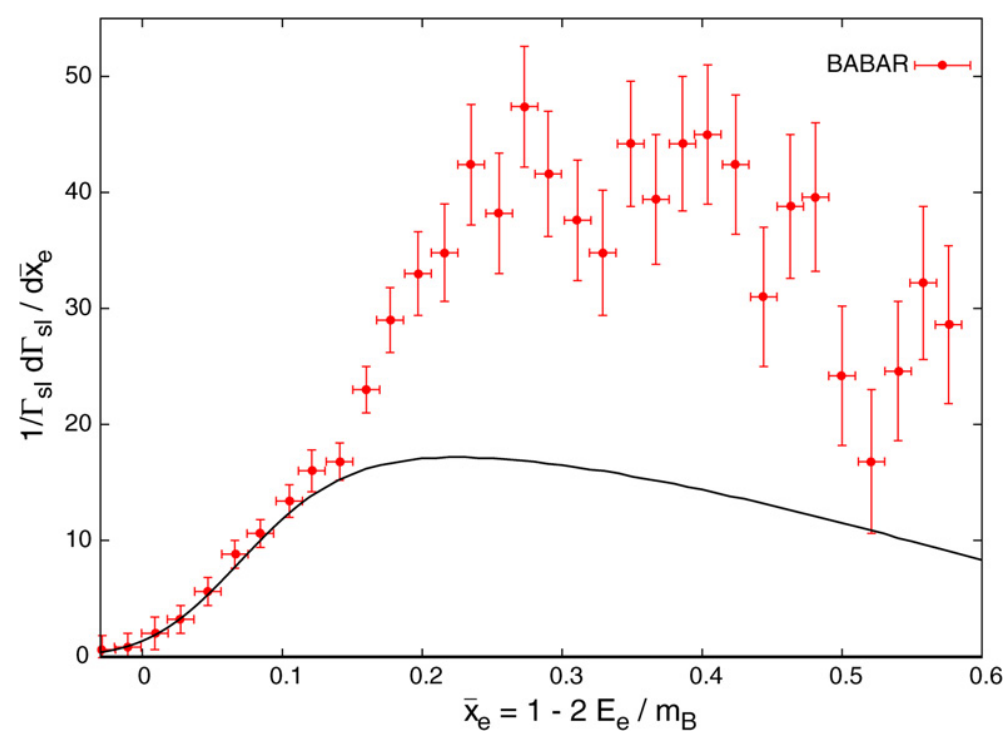

Fig. 12. Electron spectrum in semileptonic decay extending down to $1.1 \mathrm{GeV}$ from BaBar (preliminary, error bars indicate statistical errors only) compared to our model with $\alpha_{S}\left(m_{Z}\right)=0.119$. The data and the theory are normalized to one in the charm background free region $0<\bar{x}_{e}<0.125$.

We may summarize our analysis of the electron spectra by saying that the agreement theorydata is less clear in this case. The agreement is acceptable in the charm background free region, i.e. for $2.31<E_{e}<2.64 \mathrm{GeV}$; the errors in the measure of $\alpha_{S}\left(m_{Z}\right)$ are however larger than in previous cases. There is not a good agreement with the preliminary BaBar spectrum for small electron energies: our model predicts a broad maximum around $E_{e}=2.1 \mathrm{GeV}$, while the data seem to peak at lower energies.

\section{Conclusions}

We have presented a model for the QCD form factor describing radiative and semileptonic $B$ decay spectra based on soft-gluon resummation to next-to-next-to-leading logarithmic accuracy and on a power expansion in an ghost-less time-like coupling. The latter is free from infrared singularities (Landau ghost) and resumes absorptive effects in gluon cascades to all orders.

The agreement with invariant hadron mass distributions in radiative and semi-leptonic decays measured by CLEO, BaBar and Belle is in general a good one. The $\chi^{2} /$ d.o.f. values are acceptable and the extracted values of $\alpha_{S}\left(m_{Z}\right)$ are in agreement with the current PDG average within two at most standard deviations.

The agreement with the electron spectra in semi-leptonic decays is in general not as good. Even restricting the analysis to the end-point region free from the charm background (2.31$2.64 \mathrm{GeV}), \chi^{2} /$ d.o.f. values are larger and the extracted values of $\alpha_{S}\left(m_{Z}\right)$ are generally less accurate than in previous cases. The preliminary BaBar measure of the electron spectrum down to $1.1 \mathrm{GeV}$ is not in good agreement with our model, which predicts a harder spectrum, with a broad maximum around $2.1 \mathrm{GeV}$. We do not know whether the discrepancy is to be attributed to a deficiency of our model or to an under-subtracted charm background. In the former case, one could think of a non-perturbative component which is accidentally larger in the electron spectrum than in other semi-leptonic or radiative spectra. 
In general, the model seems to work quite well, validating the idea that Fermi-motion effects can be described in a resummed pQCD framework with an effective QCD coupling, which remains reasonably smaller than one in the relevant integration domain. Since the effective coupling is constructed by means of an extrapolation at low energy of the standard coupling, non-perturbative Fermi-motion effects are connected in a smooth way to the perturbative onesnamely soft gluon radiation-in our model. More accurate data on any distribution sensitive to soft-gluon effects are needed to put the model to a stringent test. Theoretical predictions could be sharpened in the future by including second-order corrections to the coefficient functions and remainder functions, as soon as they become available; that would allow to work within a complete NNLO approximation.

We have found that the inclusion of the NNLO effects in our model is crucial for a good description of the data; the model could be improved by including NNNLO terms. We have also found that the non-power expansion proposed in [1,13] does not accurately describe soft-gluon effects.

Let us end with a general comment. We find it remarkable that with such a simple model as the one we have formulated, without any adjustable parameter, it is possible to extract reasonable values of $\alpha_{S}\left(m_{Z}\right)$ from spectra with a hard scale of just a few GeV.

\section{Acknowledgements}

We would like to thank G. D’Agostini, R. Faccini and M. Pierini for discussions.

\section{Appendix A. Coefficient functions and remainder functions for the radiative decay}

The coefficient functions $C_{i}$ of the local operators $O_{i}$ entering the effective Hamiltonian

$$
\mathcal{H}^{b \rightarrow s \gamma}=\sum_{i=1}^{8} C_{i} O_{i}
$$

can be taken as:

$$
\begin{aligned}
& \left(C_{1}, C_{2}, C_{3}, C_{4}, C_{5}, C_{6}, C_{7}, C_{8}\right) \\
& \quad=(-0.480,1.023,-0.0045,-0.0640,0.0004,0.0009,-0.304,-0.148) .
\end{aligned}
$$

The analytic expressions of the $C_{i}$ 's as functions of $m_{b}, m_{t}, m_{W}$ and $\alpha_{S}\left(m_{Z}\right)$ as well as of the $O_{i}$ 's can be found in [29]. ${ }^{17}$ The coefficients $C_{i}$ for $i=3,4,5,6$ are very small, implying that the contributions of the related operators can be neglected.

The functions entering the leading-order remainder function $D_{r}^{(1)}(t)$ read in our conventions:

$$
\begin{aligned}
& f_{11}(t)=+\frac{1}{36} f_{22}(t) ; \\
& f_{12}(t)=-\frac{1}{3} f_{22}(t) ; \\
& f_{17}(t)=-\frac{1}{6} f_{27}(t) ;
\end{aligned}
$$

17 Unlike previous works ([14] and [15]), we always insert in the formulas the corrected $C_{7}=C_{7}^{(0)}+\alpha_{S} /(4 \pi) C_{7}^{(1)}$. 


$$
\begin{aligned}
& f_{18}(t)=-\frac{1}{6} f_{28}(t) \\
& f_{22}(t)=+\frac{16}{27} k\left\{t \int_{0}^{(1-t) / k} d v(1-k v)\left|\frac{G(v)}{v}+\frac{1}{2}\right|^{2}+\int_{(1-t) / k}^{1 / k} d v(1-k v)^{2}\left|\frac{G(v)}{v}+\frac{1}{2}\right|^{2}\right\} \\
& f_{27}(t)=-\frac{8}{9} k^{2}\left\{t \int_{0}^{(1-t) / k} d v \operatorname{Re}\left[G(v)+\frac{v}{2}\right]+\int_{(1-t) / k}^{1 / k} d v(1-k v) \operatorname{Re}\left[G(v)+\frac{v}{2}\right]\right\} \\
& f_{28}(t)=-\frac{1}{3} f_{27}(t) ; \\
& f_{77}(t)=+\frac{t}{9}\left[30+3 t-2 t^{2}-3(4-t) \log t\right] ; \\
& f_{78}(t)=-\frac{2}{27}\left[2 \pi^{2}-27 t+3 t^{2}-t^{3}+12 t \log t-12 \operatorname{Li}_{2}(1-t)\right] ; \\
& f_{88}(t)=+\frac{1}{81}\left\{-2 \pi^{2}+t[21+(9-2 t) t]+24 \log (1-t)\right. \\
&\left.-6 \log \frac{m_{b}}{m_{s}}[t(2+t)+4 \log (1-t)]-3 t(2+t) \log t+12 \operatorname{Li}_{2}(1-t)\right\}
\end{aligned}
$$

We have defined:

$$
G(v)= \begin{cases}-2 \arctan ^{2}\left(\sqrt{\frac{v}{4-v}}\right), & v<4 \\ 2 \log ^{2}\left[\frac{\sqrt{v}+\sqrt{v-4}}{2}\right]-2 \pi i \log \left[\frac{\sqrt{v}+\sqrt{v-4}}{2}\right]-\frac{\pi^{2}}{2}, & v \geqslant 4,\end{cases}
$$

and

$$
k=\frac{m_{c}^{2}}{m_{b}^{2}}
$$

\section{Appendix B. QCD form factor}

In this appendix we tabulate the values of the QCD form factor $\sigma(u ; w)$ in our model as a function of the infrared variable

$$
u \equiv \frac{E_{X}-\sqrt{E_{X}^{2}-m_{X}^{2}}}{E_{X}+\sqrt{E_{X}^{2}-m_{X}^{2}}} \simeq \frac{m_{X}^{2}}{4 E_{X}^{2}} \quad\left(\text { for } m_{X} \ll E_{X}\right)
$$

and of the total final hadron energy

$$
w \equiv \frac{2 E_{X}}{m_{B}}
$$

for $\alpha_{S}\left(m_{Z}\right)=0.115,0.120$ and 0.125 . The hard scale in the process is

$$
Q=w m_{B} .
$$


Table 1

$\sigma(u ; w): \alpha_{S}\left(m_{Z}\right)=\mathbf{0 . 1 1 5}$

\begin{tabular}{|c|c|c|c|c|c|c|}
\hline$u$ & $w=0.10$ & $w=0.28$ & $w=0.46$ & $w=0.64$ & $w=0.82$ & $w=1.00$ \\
\hline 0.01 & $2.89 \times 10^{-6}$ & $5.094 \times 10^{-6}$ & $1.218 \times 10^{-5}$ & $1.088 \times 10^{-4}$ & $8.073 \times 10^{-4}$ & $3.825 \times 10^{-3}$ \\
\hline 0.02 & $3.063 \times 10^{-6}$ & $1.028 \times 10^{-4}$ & $3.525 \times 10^{-3}$ & $3.077 \times 10^{-2}$ & $1.344 \times 10^{-1}$ & $3.923 \times 10^{-1}$ \\
\hline 0.03 & $3.686 \times 10^{-6}$ & $3.574 \times 10^{-3}$ & $6.732 \times 10^{-2}$ & $3.688 \times 10^{-1}$ & 1.12 & 2.426 \\
\hline 0.04 & $4.715 \times 10^{-5}$ & $3.068 \times 10^{-2}$ & $3.597 \times 10^{-1}$ & 1.407 & 3.295 & 5.788 \\
\hline 0.05 & $4.848 \times 10^{-4}$ & $1.277 \times 10^{-1}$ & 1.03 & 3.102 & 5.948 & 8.903 \\
\hline 0.06 & $2.577 \times 10^{-3}$ & $3.485 \times 10^{-1}$ & 2.065 & 5.029 & 8.209 & $1.082 \times 10^{1}$ \\
\hline 0.07 & $9.099 \times 10^{-3}$ & $7.256 \times 10^{-1}$ & 3.313 & 6.749 & 9.64 & $1.145 \times 10^{1}$ \\
\hline 0.08 & $2.451 \times 10^{-2}$ & 1.256 & 4.578 & 8.002 & $1.021 \times 10^{1}$ & $1.111 \times 10^{1}$ \\
\hline 0.09 & $5.449 \times 10^{-2}$ & 1.905 & 5.697 & 8.713 & $1.008 \times 10^{1}$ & $1.019 \times 10^{1}$ \\
\hline 0.10 & $1.052 \times 10^{-1}$ & 2.621 & 6.568 & 8.929 & 9.493 & 9.004 \\
\hline 0.11 & $1.821 \times 10^{-1}$ & 3.346 & 7.152 & 8.754 & 8.637 & 7.748 \\
\hline 0.12 & $2.894 \times 10^{-1}$ & 4.031 & 7.456 & 8.304 & 7.661 & 6.549 \\
\hline 0.13 & $4.296 \times 10^{-1}$ & 4.636 & 7.511 & 7.681 & 6.669 & 5.469 \\
\hline 0.14 & $6.029 \times 10^{-1}$ & 5.135 & 7.366 & 6.964 & 5.725 & 4.531 \\
\hline 0.15 & $8.075 \times 10^{-1}$ & 5.516 & 7.067 & 6.213 & 4.863 & 3.734 \\
\hline 0.16 & 1.04 & 5.776 & 6.661 & 5.472 & 4.099 & 3.065 \\
\hline 0.17 & 1.295 & 5.92 & 6.186 & 4.769 & 3.435 & 2.51 \\
\hline 0.18 & 1.568 & 5.958 & 5.673 & 4.119 & 2.864 & 2.05 \\
\hline 0.19 & 1.851 & 5.902 & 5.145 & 3.532 & 2.379 & 1.67 \\
\hline 0.20 & 2.138 & 5.769 & 4.621 & 3.008 & 1.968 & 1.356 \\
\hline 0.21 & 2.423 & 5.571 & 4.114 & 2.548 & 1.621 & 1.097 \\
\hline 0.22 & 2.7 & 5.325 & 3.635 & 2.146 & 1.329 & $8.814 \times 10^{-1}$ \\
\hline 0.23 & 2.963 & 5.042 & 3.187 & 1.798 & 1.083 & $7.027 \times 10^{-1}$ \\
\hline 0.24 & 3.208 & 4.734 & 2.776 & 1.498 & $8.765 \times 10^{-1}$ & $5.537 \times 10^{-1}$ \\
\hline 0.25 & 3.43 & 4.411 & 2.401 & 1.24 & $7.021 \times 10^{-1}$ & $4.293 \times 10^{-1}$ \\
\hline 0.26 & 3.627 & 4.081 & 2.063 & 1.019 & $5.55 \times 10^{-1}$ & $3.249 \times 10^{-1}$ \\
\hline 0.27 & 3.797 & 3.75 & 1.761 & $8.29 \times 10^{-1}$ & $4.307 \times 10^{-1}$ & $2.372 \times 10^{-1}$ \\
\hline 0.28 & 3.937 & 3.423 & 1.491 & $6.665 \times 10^{-1}$ & $3.255 \times 10^{-1}$ & $1.632 \times 10^{-1}$ \\
\hline 0.29 & 4.048 & 3.105 & 1.253 & $5.273 \times 10^{-1}$ & $2.363 \times 10^{-1}$ & $1.005 \times 10^{-1}$ \\
\hline 0.30 & 4.128 & 2.798 & 1.042 & $4.079 \times 10^{-1}$ & $1.606 \times 10^{-1}$ & $4.656 \times 10^{-2}$ \\
\hline 0.31 & 4.18 & 2.504 & $8.571 \times 10^{-1}$ & $3.057 \times 10^{-1}$ & $9.62 \times 10^{-2}$ & $1.027 \times 10^{-4}$ \\
\hline 0.32 & 4.202 & 2.227 & $6.948 \times 10^{-1}$ & $2.18 \times 10^{-1}$ & $4.139 \times 10^{-2}$ & $-3.977 \times 10^{-2}$ \\
\hline 0.33 & 4.197 & 1.965 & $5.528 \times 10^{-1}$ & $1.427 \times 10^{-1}$ & $-5.313 \times 10^{-3}$ & $-7.377 \times 10^{-2}$ \\
\hline 0.34 & 4.166 & 1.722 & $4.29 \times 10^{-1}$ & $7.814 \times 10^{-2}$ & $-4.524 \times 10^{-2}$ & $-1.026 \times 10^{-1}$ \\
\hline 0.35 & 4.112 & 1.496 & $3.211 \times 10^{-1}$ & $2.272 \times 10^{-2}$ & $-7.955 \times 10^{-2}$ & $-1.27 \times 10^{-1}$ \\
\hline 0.36 & 4.035 & 1.287 & $2.274 \times 10^{-1}$ & $-2.485 \times 10^{-2}$ & $-1.093 \times 10^{-1}$ & $-1.476 \times 10^{-1}$ \\
\hline 0.37 & 3.938 & 1.096 & $1.463 \times 10^{-1}$ & $-6.568 \times 10^{-2}$ & $-1.353 \times 10^{-1}$ & $-1.649 \times 10^{-1}$ \\
\hline 0.38 & 3.824 & $9.216 \times 10^{-1}$ & $7.613 \times 10^{-2}$ & $-1.007 \times 10^{-1}$ & $-1.578 \times 10^{-1}$ & $-1.796 \times 10^{-1}$ \\
\hline 0.39 & 3.693 & $7.63 \times 10^{-1}$ & $1.552 \times 10^{-2}$ & $-1.307 \times 10^{-1}$ & $-1.771 \times 10^{-1}$ & $-1.918 \times 10^{-1}$ \\
\hline 0.40 & 3.55 & $6.195 \times 10^{-1}$ & $-3.678 \times 10^{-2}$ & $-1.564 \times 10^{-1}$ & $-1.936 \times 10^{-1}$ & $-2.021 \times 10^{-1}$ \\
\hline 0.50 & 1.791 & $-1.934 \times 10^{-1}$ & $-2.85 \times 10^{-1}$ & $-2.775 \times 10^{-1}$ & $-2.596 \times 10^{-1}$ & $-2.42 \times 10^{-1}$ \\
\hline 0.60 & $3.035 \times 10^{-1}$ & $-3.989 \times 10^{-1}$ & $-3.229 \times 10^{-1}$ & $-2.851 \times 10^{-1}$ & $-2.551 \times 10^{-1}$ & $-2.346 \times 10^{-1}$ \\
\hline 0.70 & $-4.713 \times 10^{-1}$ & $-4.072 \times 10^{-1}$ & $-3.088 \times 10^{-1}$ & $-2.615 \times 10^{-1}$ & $-2.335 \times 10^{-1}$ & $-2.15 \times 10^{-1}$ \\
\hline 0.80 & $-7.243 \times 10^{-1}$ & $-3.596 \times 10^{-1}$ & $-2.718 \times 10^{-1}$ & $-2.311 \times 10^{-1}$ & $-2.076 \times 10^{-1}$ & $-1.92 \times 10^{-1}$ \\
\hline 0.90 & $-6.43 \times 10^{-1}$ & $-2.953 \times 10^{-1}$ & $-2.286 \times 10^{-1}$ & $-1.975 \times 10^{-1}$ & $-1.792 \times 10^{-1}$ & $-1.653 \times 10^{-1}$ \\
\hline 0.98 & $-4.249 \times 10^{-1}$ & $-2.458 \times 10^{-1}$ & $-2.017 \times 10^{-1}$ & $-1.803 \times 10^{-1}$ & $-1.672 \times 10^{-1}$ & $-1.58 \times 10^{-1}$ \\
\hline
\end{tabular}


Table 2

$\sigma(u ; w): \alpha_{S}\left(m_{Z}\right)=\mathbf{0 . 1 2 0}$

\begin{tabular}{|c|c|c|c|c|c|c|}
\hline$u$ & $w=0.10$ & $w=0.28$ & $w=0.46$ & $w=0.64$ & $w=0.82$ & $w=1.00$ \\
\hline 0.01 & $2.575 \times 10^{-6}$ & $4.491 \times 10^{-6}$ & $6.691 \times 10^{-6}$ & $2.153 \times 10^{-5}$ & $1.321 \times 10^{-4}$ & $6.542 \times 10^{-4}$ \\
\hline 0.02 & $2.748 \times 10^{-6}$ & $2.286 \times 10^{-5}$ & $7.371 \times 10^{-4}$ & $7.231 \times 10^{-3}$ & $3.539 \times 10^{-2}$ & $1.147 \times 10^{-1}$ \\
\hline 0.03 & $2.992 \times 10^{-6}$ & $8.639 \times 10^{-4}$ & $1.881 \times 10^{-2}$ & $1.185 \times 10^{-1}$ & $4.081 \times 10^{-1}$ & $9.901 \times 10^{-1}$ \\
\hline 0.04 & $1.341 \times 10^{-5}$ & $8.992 \times 10^{-3}$ & $1.247 \times 10^{-1}$ & $5.674 \times 10^{-1}$ & 1.517 & 2.995 \\
\hline 0.05 & $1.359 \times 10^{-4}$ & $4.372 \times 10^{-2}$ & $4.23 \times 10^{-1}$ & 1.493 & 3.282 & 5.527 \\
\hline 0.06 & $8.021 \times 10^{-4}$ & $1.357 \times 10^{-1}$ & $9.753 \times 10^{-1}$ & 2.797 & 5.245 & 7.775 \\
\hline 0.07 & $3.121 \times 10^{-3}$ & $3.155 \times 10^{-1}$ & 1.761 & 4.239 & 6.961 & 9.285 \\
\hline 0.08 & $9.146 \times 10^{-3}$ & $6.013 \times 10^{-1}$ & 2.695 & 5.582 & 8.182 & 9.98 \\
\hline 0.09 & $2.192 \times 10^{-2}$ & $9.932 \times 10^{-1}$ & 3.67 & 6.663 & 8.849 & 9.991 \\
\hline 0.10 & $4.521 \times 10^{-2}$ & 1.475 & 4.587 & 7.407 & 9.021 & 9.516 \\
\hline 0.11 & $8.315 \times 10^{-2}$ & 2.018 & 5.373 & 7.812 & 8.805 & 8.74 \\
\hline 0.12 & $1.397 \times 10^{-1}$ & 2.589 & 5.986 & 7.916 & 8.316 & 7.817 \\
\hline 0.13 & $2.181 \times 10^{-1}$ & 3.157 & 6.41 & 7.774 & 7.657 & 6.855 \\
\hline 0.14 & $3.208 \times 10^{-1}$ & 3.692 & 6.65 & 7.447 & 6.911 & 5.924 \\
\hline 0.15 & $4.489 \times 10^{-1}$ & 4.171 & 6.724 & 6.991 & 6.141 & 5.062 \\
\hline 0.16 & $6.024 \times 10^{-1}$ & 4.58 & 6.657 & 6.453 & 5.388 & 4.29 \\
\hline 0.17 & $7.799 \times 10^{-1}$ & 4.909 & 6.474 & 5.873 & 4.681 & 3.612 \\
\hline 0.18 & $9.791 \times 10^{-1}$ & 5.155 & 6.201 & 5.282 & 4.033 & 3.024 \\
\hline 0.19 & 1.197 & 5.317 & 5.862 & 4.702 & 3.451 & 2.521 \\
\hline 0.20 & 1.429 & 5.401 & 5.478 & 4.149 & 2.935 & 2.093 \\
\hline 0.21 & 1.671 & 5.413 & 5.067 & 3.633 & 2.482 & 1.73 \\
\hline 0.22 & 1.918 & 5.361 & 4.643 & 3.159 & 2.089 & 1.423 \\
\hline 0.23 & 2.167 & 5.254 & 4.219 & 2.729 & 1.748 & 1.163 \\
\hline 0.24 & 2.412 & 5.1 & 3.803 & 2.343 & 1.454 & $9.444 \times 10^{-1}$ \\
\hline 0.25 & 2.649 & 4.909 & 3.404 & 1.999 & 1.201 & $7.596 \times 10^{-1}$ \\
\hline 0.26 & 2.875 & 4.689 & 3.025 & 1.695 & $9.845 \times 10^{-1}$ & $6.034 \times 10^{-1}$ \\
\hline 0.27 & 3.085 & 4.446 & 2.67 & 1.427 & $7.986 \times 10^{-1}$ & $4.712 \times 10^{-1}$ \\
\hline 0.28 & 3.278 & 4.186 & 2.34 & 1.193 & $6.394 \times 10^{-1}$ & $3.593 \times 10^{-1}$ \\
\hline 0.29 & 3.451 & 3.916 & 2.037 & $9.873 \times 10^{-1}$ & $5.031 \times 10^{-1}$ & $2.641 \times 10^{-1}$ \\
\hline 0.30 & 3.602 & 3.64 & 1.761 & $8.082 \times 10^{-1}$ & $3.864 \times 10^{-1}$ & $1.828 \times 10^{-1}$ \\
\hline 0.31 & 3.73 & 3.362 & 1.51 & $6.522 \times 10^{-1}$ & $2.864 \times 10^{-1}$ & $1.127 \times 10^{-1}$ \\
\hline 0.32 & 3.834 & 3.086 & 1.283 & $5.165 \times 10^{-1}$ & $2.008 \times 10^{-1}$ & $5.236 \times 10^{-2}$ \\
\hline 0.33 & 3.913 & 2.815 & 1.08 & $3.986 \times 10^{-1}$ & $1.274 \times 10^{-1}$ & $5.426 \times 10^{-4}$ \\
\hline 0.34 & 3.968 & 2.552 & $8.978 \times 10^{-1}$ & $2.963 \times 10^{-1}$ & $6.449 \times 10^{-2}$ & $-4.377 \times 10^{-2}$ \\
\hline 0.35 & 3.999 & 2.298 & $7.355 \times 10^{-1}$ & $2.076 \times 10^{-1}$ & $1.037 \times 10^{-2}$ & $-8.151 \times 10^{-2}$ \\
\hline 0.36 & 4.007 & 2.055 & $5.915 \times 10^{-1}$ & $1.308 \times 10^{-1}$ & $-3.641 \times 10^{-2}$ & $-1.136 \times 10^{-1}$ \\
\hline 0.37 & 3.992 & 1.824 & $4.64 \times 10^{-1}$ & $6.426 \times 10^{-2}$ & $-7.71 \times 10^{-2}$ & $-1.407 \times 10^{-1}$ \\
\hline 0.38 & 3.956 & 1.607 & $3.515 \times 10^{-1}$ & $6.723 \times 10^{-3}$ & $-1.125 \times 10^{-1}$ & $-1.636 \times 10^{-1}$ \\
\hline 0.39 & 3.9 & 1.403 & $2.527 \times 10^{-1}$ & $-4.299 \times 10^{-2}$ & $-1.433 \times 10^{-1}$ & $-1.83 \times 10^{-1}$ \\
\hline 0.40 & 3.826 & 1.212 & $1.659 \times 10^{-1}$ & $-8.59 \times 10^{-2}$ & $-1.697 \times 10^{-1}$ & $-1.993 \times 10^{-1}$ \\
\hline 0.50 & 2.42 & $-9.317 \times 10^{-3}$ & $-2.727 \times 10^{-1}$ & $-2.938 \times 10^{-1}$ & $-2.836 \times 10^{-1}$ & $-2.655 \times 10^{-1}$ \\
\hline 0.60 & $7.765 \times 10^{-1}$ & $-4.072 \times 10^{-1}$ & $-3.595 \times 10^{-1}$ & $-3.213 \times 10^{-1}$ & $-2.858 \times 10^{-1}$ & $-2.61 \times 10^{-1}$ \\
\hline 0.70 & $-3.087 \times 10^{-1}$ & $-4.693 \times 10^{-1}$ & $-3.545 \times 10^{-1}$ & $-2.965 \times 10^{-1}$ & $-2.617 \times 10^{-1}$ & $-2.388 \times 10^{-1}$ \\
\hline 0.80 & $-7.857 \times 10^{-1}$ & $-4.228 \times 10^{-1}$ & $-3.12 \times 10^{-1}$ & $-2.604 \times 10^{-1}$ & $-2.312 \times 10^{-1}$ & $-2.12 \times 10^{-1}$ \\
\hline 0.90 & $-7.863 \times 10^{-1}$ & $-3.432 \times 10^{-1}$ & $-2.588 \times 10^{-1}$ & $-2.199 \times 10^{-1}$ & $-1.975 \times 10^{-1}$ & $-1.808 \times 10^{-1}$ \\
\hline 0.98 & $-5.003 \times 10^{-1}$ & $-2.753 \times 10^{-1}$ & $-2.213 \times 10^{-1}$ & $-1.96 \times 10^{-1}$ & $-1.805 \times 10^{-1}$ & $-1.699 \times 10^{-1}$ \\
\hline
\end{tabular}


Table 3

$\sigma(u ; w): \alpha_{S}\left(m_{Z}\right)=\mathbf{0 . 1 2 5}$

\begin{tabular}{|c|c|c|c|c|c|c|}
\hline$u$ & $w=0.10$ & $w=0.28$ & $w=0.46$ & $w=0.64$ & $w=0.82$ & $w=1.00$ \\
\hline 0.01 & $2.309 \times 10^{-6}$ & $4.039 \times 10^{-6}$ & $5.47 \times 10^{-6}$ & $8.764 \times 10^{-6}$ & $2.845 \times 10^{-5}$ & $1.247 \times 10^{-4}$ \\
\hline 0.02 & $2.46 \times 10^{-6}$ & $8.166 \times 10^{-6}$ & $1.706 \times 10^{-4}$ & $1.785 \times 10^{-3}$ & $9.505 \times 10^{-3}$ & $3.339 \times 10^{-2}$ \\
\hline 0.03 & $2.641 \times 10^{-6}$ & $2.309 \times 10^{-4}$ & $5.49 \times 10^{-3}$ & $3.839 \times 10^{-2}$ & $1.458 \times 10^{-1}$ & $3.871 \times 10^{-1}$ \\
\hline 0.04 & $5.702 \times 10^{-6}$ & $2.809 \times 10^{-3}$ & $4.39 \times 10^{-2}$ & $2.243 \times 10^{-1}$ & $6.666 \times 10^{-1}$ & 1.448 \\
\hline 0.05 & $4.474 \times 10^{-5}$ & $1.562 \times 10^{-2}$ & $1.727 \times 10^{-1}$ & $6.899 \times 10^{-1}$ & 1.695 & 3.153 \\
\hline 0.06 & $2.796 \times 10^{-4}$ & $5.422 \times 10^{-2}$ & $4.501 \times 10^{-1}$ & 1.47 & 3.092 & 5.072 \\
\hline 0.07 & $1.174 \times 10^{-3}$ & $1.387 \times 10^{-1}$ & $9.02 \times 10^{-1}$ & 2.485 & 4.589 & 6.778 \\
\hline 0.08 & $3.689 \times 10^{-3}$ & $2.875 \times 10^{-1}$ & 1.512 & 3.596 & 5.938 & 8.023 \\
\hline 0.09 & $9.412 \times 10^{-3}$ & $5.117 \times 10^{-1}$ & 2.231 & 4.665 & 6.988 & 8.735 \\
\hline 0.10 & $2.055 \times 10^{-2}$ & $8.124 \times 10^{-1}$ & 2.996 & 5.587 & 7.678 & 8.96 \\
\hline 0.11 & $3.98 \times 10^{-2}$ & 1.181 & 3.745 & 6.303 & 8.017 & 8.791 \\
\hline 0.12 & $7.011 \times 10^{-2}$ & 1.603 & 4.429 & 6.79 & 8.05 & 8.34 \\
\hline 0.13 & $1.144 \times 10^{-1}$ & 2.057 & 5.012 & 7.056 & 7.838 & 7.71 \\
\hline 0.14 & $1.752 \times 10^{-1}$ & 2.524 & 5.473 & 7.121 & 7.447 & 6.985 \\
\hline 0.15 & $2.547 \times 10^{-1}$ & 2.982 & 5.805 & 7.019 & 6.935 & 6.228 \\
\hline 0.16 & $3.542 \times 10^{-1}$ & 3.415 & 6.01 & 6.781 & 6.354 & 5.482 \\
\hline 0.17 & $4.742 \times 10^{-1}$ & 3.809 & 6.098 & 6.443 & 5.743 & 4.777 \\
\hline 0.18 & $6.145 \times 10^{-1}$ & 4.152 & 6.082 & 6.034 & 5.133 & 4.127 \\
\hline 0.19 & $7.741 \times 10^{-1}$ & 4.439 & 5.975 & 5.582 & 4.544 & 3.54 \\
\hline 0.20 & $9.512 \times 10^{-1}$ & 4.666 & 5.794 & 5.108 & 3.99 & 3.017 \\
\hline 0.21 & 1.143 & 4.832 & 5.554 & 4.63 & 3.477 & 2.558 \\
\hline 0.22 & 1.348 & 4.939 & 5.268 & 4.161 & 3.011 & 2.156 \\
\hline 0.23 & 1.561 & 4.99 & 4.95 & 3.71 & 2.591 & 1.808 \\
\hline 0.24 & 1.781 & 4.989 & 4.611 & 3.285 & 2.216 & 1.507 \\
\hline 0.25 & 2.002 & 4.942 & 4.26 & 2.889 & 1.883 & 1.247 \\
\hline 0.26 & 2.223 & 4.855 & 3.907 & 2.525 & 1.59 & 1.024 \\
\hline 0.27 & 2.438 & 4.731 & 3.558 & 2.192 & 1.333 & $8.328 \times 10^{-1}$ \\
\hline 0.28 & 2.647 & 4.577 & 3.217 & 1.891 & 1.108 & $6.686 \times 10^{-1}$ \\
\hline 0.29 & 2.844 & 4.399 & 2.89 & 1.619 & $9.118 \times 10^{-1}$ & $5.278 \times 10^{-1}$ \\
\hline 0.30 & 3.029 & 4.199 & 2.579 & 1.376 & $7.41 \times 10^{-1}$ & $4.067 \times 10^{-1}$ \\
\hline 0.31 & 3.199 & 3.984 & 2.286 & 1.159 & $5.926 \times 10^{-1}$ & $3.022 \times 10^{-1}$ \\
\hline 0.32 & 3.352 & 3.757 & 2.012 & $9.665 \times 10^{-1}$ & $4.639 \times 10^{-1}$ & $2.117 \times 10^{-1}$ \\
\hline 0.33 & 3.486 & 3.522 & 1.758 & $7.956 \times 10^{-1}$ & $3.523 \times 10^{-1}$ & $1.334 \times 10^{-1}$ \\
\hline 0.34 & 3.601 & 3.282 & 1.523 & $6.446 \times 10^{-1}$ & $2.557 \times 10^{-1}$ & $6.584 \times 10^{-2}$ \\
\hline 0.35 & 3.696 & 3.04 & 1.308 & $5.114 \times 10^{-1}$ & $1.719 \times 10^{-1}$ & $7.763 \times 10^{-3}$ \\
\hline 0.36 & 3.769 & 2.8 & 1.112 & $3.943 \times 10^{-1}$ & $9.927 \times 10^{-2}$ & $-4.2 \times 10^{-2}$ \\
\hline 0.37 & 3.822 & 2.563 & $9.342 \times 10^{-1}$ & $2.914 \times 10^{-1}$ & $3.593 \times 10^{-2}$ & $-8.451 \times 10^{-2}$ \\
\hline 0.38 & 3.855 & 2.331 & $7.732 \times 10^{-1}$ & $2.013 \times 10^{-1}$ & $-1.942 \times 10^{-2}$ & $-1.207 \times 10^{-1}$ \\
\hline 0.39 & 3.866 & 2.106 & $6.284 \times 10^{-1}$ & $1.224 \times 10^{-1}$ & $-6.775 \times 10^{-2}$ & $-1.515 \times 10^{-1}$ \\
\hline 0.40 & 3.858 & 1.89 & $4.984 \times 10^{-1}$ & $5.351 \times 10^{-2}$ & $-1.098 \times 10^{-1}$ & $-1.775 \times 10^{-1}$ \\
\hline 0.50 & 2.917 & $2.99 \times 10^{-1}$ & $-2.15 \times 10^{-1}$ & $-2.945 \times 10^{-1}$ & $-3.031 \times 10^{-1}$ & $-2.883 \times 10^{-1}$ \\
\hline 0.60 & 1.277 & $-3.637 \times 10^{-1}$ & $-3.894 \times 10^{-1}$ & $-3.592 \times 10^{-1}$ & $-3.194 \times 10^{-1}$ & $-2.902 \times 10^{-1}$ \\
\hline 0.70 & $-6.465 \times 10^{-2}$ & $-5.265 \times 10^{-1}$ & $-4.05 \times 10^{-1}$ & $-3.365 \times 10^{-1}$ & $-2.936 \times 10^{-1}$ & $-2.656 \times 10^{-1}$ \\
\hline 0.80 & $-7.947 \times 10^{-1}$ & $-4.951 \times 10^{-1}$ & $-3.589 \times 10^{-1}$ & $-2.94 \times 10^{-1}$ & $-2.578 \times 10^{-1}$ & $-2.344 \times 10^{-1}$ \\
\hline 0.90 & $-9.333 \times 10^{-1}$ & $-3.996 \times 10^{-1}$ & $-2.936 \times 10^{-1}$ & $-2.452 \times 10^{-1}$ & $-2.179 \times 10^{-1}$ & $-1.978 \times 10^{-1}$ \\
\hline 0.98 & $-5.886 \times 10^{-1}$ & $-3.088 \times 10^{-1}$ & $-2.428 \times 10^{-1}$ & $-2.128 \times 10^{-1}$ & $-1.947 \times 10^{-1}$ & $-1.826 \times 10^{-1}$ \\
\hline
\end{tabular}


In the radiative case one sets $t=u$ and $w=1$ while in the semileptonic case the form factor as a function of $w(0<w<1)$ is needed [9,20,21]. By using Tables 1, 2 and 3, the reader can obtain the form factor for all the values of $u$ and $w$ by means of a straightforward interpolation, avoiding the delicate numerical integrations related to the Mellin transform and to the inverse Mellin transform. In agreement with physical intuition, by lowering the hard scale, the peak of the form factor broadens and shifts to larger $u$ 's because of the coupling growth.

\section{References}

[1] For a review of the theoretical activity, see for example: D.V. Shirkov, Nucl. Phys. B (Proc. Suppl.) 152 (2006) 51, hep-ph/0408272.

[2] I.I.Y. Bigi, M.A. Shifman, N.G. Uraltsev, A.I. Vainshtein, Int. J. Mod. Phys. A 9 (1994) 2467, hep-ph/9312359.

[3] For a recent analysis see for example: O. Buchmuller, H. Flacher, Phys. Rev. D 73 (2006) 073008, hep-ph/0507253.

[4] S. Catani, M.L. Mangano, P. Nason, L. Trentadue, Nucl. Phys. B 478 (1996) 273, hep-ph/9604351.

[5] A similar philosophy has been applied to the description of $e^{+} e^{-}$shape variables in: Y.L. Dokshitzer, G. Marchesini, B.R. Webber, Nucl. Phys. B 469 (1996) 93, hep-ph/9512336;

The main difference is that we do not have free parameters. A similar philosophy has also been exploited in the frame of the renormalon calculus in: J.R. Andersen, E. Gardi, JHEP 0601 (2006) 097, hep-ph/0509360;

The main difference is that we consider a set of summable corrections by means of the analyticity principle.

[6] For a discussion of the kinematics involved in the renormalization of the QCD coupling, see for example: Y.L. Dokshitzer, V.A. Khoze, A.H. Mueller, S.I. Troian, Basics of Perturbative QCD, Editions Frontieres, Paris, 1991, Chapter one.

[7] U. Aglietti, Z. Ligeti, Phys. Lett. B 364 (1995) 75, hep-ph/9503209.

[8] See for example: L. Landau, E. Lifshitz, Teoria Quantistica Relativistica, vol. 4, Editori Riuniti, 1978, last chapter.

[9] U. Aglietti, G. Ricciardi, G. Ferrera, Phys. Rev. D 74 (2006) 034004, hep-ph/0507285.

[10] A.Y. Alekseev, Phys. Rev. D 61 (2000) 114005; A.I. Alekseev, hep-ph/0011178.

[11] D. Amati, A. Bassetto, M. Ciafaloni, G. Marchesini, G. Veneziano, Nucl. Phys. B 173 (1980) 429.

[12] S. Moch, J.A.M. Vermaseren, A. Vogt, Nucl. Phys. B 726 (2005) 317, hep-ph/0506288.

[13] U. Aglietti, G. Ricciardi, Phys. Rev. D 70 (2004) 114008, hep-ph/0407225.

[14] U. Aglietti, Nucl. Phys. B 610 (2001) 293, hep-ph/0104020.

[15] U. Aglietti, M. Ciuchini, P. Gambino, Nucl. Phys. B 637 (2002) 427, hep-ph/0204140.

[16] B. Aubert, et al., BaBar Collaboration, Phys. Rev. D 72 (2005) 052004, hep-ex/0508004.

[17] S. Chen, et al., CLEO Collaboration, Phys. Rev. Lett. 87 (2001) 251807, hep-ex/0108032.

[18] B. Aubert, et al., BaBar Collaboration, hep-ex/0507001.

[19] P. Koppenburg, et al., Belle Collaboration, Phys. Rev. Lett. 93 (2004) 061803, hep-ex/0403004.

[20] U. Aglietti, G. Ricciardi, G. Ferrera, Phys. Rev. D 74 (2006) 034005, hep-ph/0509095.

[21] U. Aglietti, G. Ricciardi, G. Ferrera, Phys. Rev. D 74 (2006) 034006, hep-ph/0509271.

[22] B.O. Lange, M. Neubert, G. Paz, Phys. Rev. D 72 (2005) 073006, hep-ph/0504071.

[23] B. Aubert, et al., BaBar Collaboration, Phys. Rev. Lett. 92 (2004) 071802, hep-ex/0307062.

[24] I. Bizjak, et al., Belle Collaboration, Phys. Rev. Lett. 95 (2005) 241801, hep-ex/0505088.

[25] A. Bornheim, et al., CLEO Collaboration, Phys. Rev. Lett. 88 (2002) 231803, hep-ex/0202019.

[26] B. Aubert, et al., BaBar Collaboration, Phys. Rev. D 73 (2006) 012006, hep-ex/0509040.

[27] A. Limosani, et al., Belle Collaboration, Phys. Lett. B 621 (2005) 28, hep-ex/0504046.

[28] B. Aubert, et al., BaBar Collaboration, hep-ex/0408075.

[29] K.G. Chetyrkin, M. Misiak, M. Munz, Phys. Lett. B 400 (1997) 206, hep-ph/9612313;

K.G. Chetyrkin, M. Misiak, M. Munz, Phys. Lett. B 425 (1998) 414, Erratum;

P. Gambino, M. Gorbahn, U. Haisch, Nucl. Phys. B 673 (2003) 238, hep-ph/0306079.

[30] W.-M. Yao, et al., J. Phys. G 33 (2006) 1. 